\title{
Direct pore-to-core up-scaling of displacement processes: Experimentation and dynamic pore network modeling
}

\author{
Arash Aghaei ${ }^{a}$, Mohammad Piri ${ }^{\mathrm{a}, *}$ \\ ${ }^{a}$ Department of Chemical and Petroleum Engineering, University of Wyoming, Laramie, \\ Wyoming, USA.
}

\begin{abstract}
We present a new dynamic pore network model that is capable of up-scaling two-phase flow processes from pore to core. This dynamic model provides a platform to study various flow processes in porous media at the core scale using the pore-scale physics. The most critical features of this platform include (1) the incorporation of viscous, capillary, and gravity pressure drops in pore-scale displacement thresholds, (2) wetting-phase corner flow in capillary elements with angular cross-sections, (3) adjustments of corner interfaces between wetting and non-wetting phases based on changes in local capillary pressure, (4) simultaneous injection of wetting and non-wetting phases from the inlet of the medium at constant flow rates that makes the study of steadystate processes possible, (5) heavy parallelization using a three-dimensional domain decomposition scheme that enables the study of two-phase flow at the core scale, and (6) constant pressure boundary condition at the outlet. For the validation of the dynamic model, three two-phase miniature core-flooding experiments were performed in a state-of-the-art micro core-flooding system
\end{abstract}

${ }^{*}$ Corresponding author. E-mail address: mpiri@uwyo.edu.

Preprint submitted to Journal of Hydrology

January 7, 2015

(C) 2015. This manuscript version is made available under the Elsevier user license http://www.elsevier.com/open-access/userlicense/1.0/ 
integrated with a high-resolution X-ray micro-CT scanner. The dynamic model was rigorously validated by comparing the predicted local saturation profiles, fractional flow curves, relative permeabilities, and residual oil saturations against their experimental counterparts. The validated dynamic model was then used to study low-IFT and high viscosity two-phase flow processes and investigate the effect of high capillary number on relative permeabilities and residual oil saturation.

Keywords:

Up-scaling, Pore-scale flows, Pore network modeling, Microtomography, Core-flooding experiments

\section{1. Introduction}

2 Multiphase flow in porous media occurs in many natural and artificial processes such as subsurface flow of hydrocarbons and brine, geologic storage of ${ }_{4} \mathrm{CO}_{2}$, non-aqueous phase liquids (NAPL) migration in soil, reactive trans5 port, and water removal in gas diffusion layer (GDL) of proton exchange 6 membrane (PEM) fuel cells. Understanding the displacement and transport 7 processes relevant to multiphase flow systems and predicting the associated 8 macroscopic properties are crucial for design and prediction of performance 9 of these processes (Dullien, 1992; Sahimi, 2010).

10 In petroleum and environmental engineering contexts, multiphase flow in porous rocks has been studied extensively using experimental and numerical techniques at multiple scales. Large-scale continuum models (e.g. reservoir models) generally solve mass conservation partial differential equations over 14 grid blocks of the medium that are larger than (or equal to) Representa- 
tive Elementary Volume (REV) of that medium. These models read as input multiphase flow functions such as relative permeabilities. These functions are manifestation of many pore-scale phenomena, and they inform the numerical solvers of mass conservation equations about the underlying displacement physics. Pore-scale investigations are often used to develop improved understanding of fundamental phenomena relevant to a given process and predict the pertinent flow and transport properties. These physically-based properties are then used to inform the larger-scale continuum models.

The pore-scale models can be categorized into direct and network models. In direct models, multiphase flow is simulated directly in the pore space structure that is mapped using an imaging technique or created by a processbased reconstruction method, e.g., sedimentation simulation. Direct models include Lagrangian particle-based (mesh-free) methods, such as moving particle semi-implicit (MPS) (Koshizuka et al., 1995; Premože et al., 2003; Ovaysi and Piri, 2010, 2011), smoothed particle hydrodynamics (SPH) (Gingold and Monaghan, 1997; Zhu et al., 1999; Tartakovsky and Meakin, 2005), and Lattice Boltzmann (Inamuro et al., 2004; Li et al., 2005), and meshbased methods, e.g., finite element (Fourie et al., 2007). These models use accurate representation of the pore space. However, due to irregular fluidsolid boundaries and deformability of fluid-fluid interfaces, direct models are computationally expensive and may not be suitable for studying multiphase flow at the core scale. In the second group of pore-scale models, i.e., network models, the pore space is represented by a network of idealized pores and throats. Pore-scale displacements are carried out in the pore network to simulate multiphase flow (Øren et al., 1998; Patzek, 2001; Blunt et al., 2002; 
Piri and Blunt, 2005a,b).

Pore network modeling was first introduced in the 1950's by Fatt (1956a,b,c) who used a network of real resistors to calculate relative permeability and capillary pressure for a drainage process. Network modeling, since its introduction, has evolved enormously. Today, one can map the pore space of a rock sample with high-resolution imaging techniques and extract an equivalent pore network (Dong and Blunt, 2009). Our knowledge of the pore-scale displacement physics has also improved dramatically due to micro-fluidics and other types of experiments. Pore network models can be divided into quasi-static and dynamic. The majority of the previously-developed network models are quasi-static in which the pore-scale displacements take place based on their threshold capillary pressure. These models have had significant success in modeling two- and three-phase flow in porous media under capillary-dominated conditions (Øren et al., 1998; Patzek, 2001; Øren and Bakke, 2003; Valvatne and Blunt, 2004; Piri and Blunt, 2005a,b). However, quasi-static models do not include the effects of viscous and gravity forces, and therefore, cannot be used to study cases in which capillary-dominated assumptions do not apply. Viscous and gravity forces become significant during many subsurface flow processes, such as enhanced oil recovery (EOR) methods of polymer and surfactant flooding, and high velocity flow regimes that are encountered in naturally and hydraulically induced fractures as well as near well-bore areas (Lake, 1989; Sahimi, 2010). In these cases, the combined effects of capillary, viscous, and gravity forces determine the flow behavior in porous media. In dynamic network models, on the other hand, viscous and in some cases gravity forces are taken into account. These models can be used 
to study the cases in which viscous or gravity forces are relevant. However, due to various reasons, such as difficulties in implementing the complex porescale physics and the computational costs associated with these models, the previously-developed dynamic models lack some of the critical capabilities to successfully simulate dynamic two-phase flow processes at the core scale.

Tables 1 and 2 list the previously-developed dynamic pore network models along with their largest network size, phenomena studied, and validation techniques. A comprehensive review of dynamic pore network models of twophase flow in porous media can be found elsewhere, see, Joekar-Niasar and Hassanizadeh (2012) and Aghaei (2014). Here, we discuss the characteristics and predictive capabilities of each previously-developed model. We then present an overview of the dynamic model developed under this study and explain the critical relevance of its capabilities within the context of direct up-scaling.

Koplik and Lasseter (1985) developed the first dynamic network model to study the effects of microscopic pore structure on macroscopic phenomena. They stated the computational difficulties associated with dynamic pore network modeling as the limiting constraint in selecting the network size. Lenormand et al. (1988), Blunt and King (1991), and Lee et al. (1995) developed models in which the pores contained all the fluid and the pressure drops took place exclusively in the throats. Lenormand et al. (1988) performed drainage simulations at various capillary numbers and viscosity ratios and identified three distinct flow patterns. Blunt and King (1991) ran drainage simulations in two- and three-dimensional random networks and calculated relative permeabilities based on local flow rates and pressure drops. 
The model developed by Lee et al. (1995) was a parallel model that was used to perform water-flooding and miscible-flooding simulations in networks as large as 524,288 pores. This model was later extended by Kamath et al. (1996) and $\mathrm{Xu}$ et al. (1999) who were able to to reproduce recoveries in a fully miscible core-flooding experiment in a dolomite sample.

van der Marck et al. (1997) extended the model introduced by Lenormand et al. (1988) by allowing up to two menisci in pore throats. Mogensen and Stenby (1998) developed a model in which the corner flow and snapoff displacements were incorporated. Aker et al. (1998) developed a model with hourglass-shaped throats to study time dependencies of pressure distribution and fluid front in drainage processes. Later, Knudsen and Hansen (2002) modified this model by adding biperiodic boundary conditions. Dahle and Celia (1999) introduced a new interface tracking method in which fluids could form compartments that were separated by fluid-fluid interfaces. Hughes and Blunt (2000) used the wetting-phase viscous pressure drop to perturb the order of displacements. This quasi-dynamic model was used to study the effect of the capillary number during imbibition. Constantinides and Payatakes (2000) studied the effects of wetting layers on disconnection of the non-wetting phase during imbibition. Thompson (2002) created random pore networks with converging-diverging geometry for throats to study drainage and imbibition in fibrous materials. Singh and Mohanty (2003) introduced a new method for handling the corner flow in which the wetting phase was removed from the layers in proportion to the local capillary pressure drop. Nordhaug et al. (2003) extended the model developed by Blunt and King (1991) to study interfacial velocities and areas. Løvoll et al. (2005) 
studied drainage of a high-viscosity wetting phase and stabilizing effect of gravity using a dynamic pore network model and glass beads experiments.

Al-Gharbi and Blunt (2005) incorporated layer swelling and snap-off displacements in a dynamic model and used it to study the effects of capillary number and viscosity ratio in drainage in 2D lattices. Nguyen et al. (2006) introduced a new network model to study the competition between snap-off and piston-like displacements during imbibition and its effect on relative permeabilities and residual oil saturation. The only dynamic effect included in this model was the viscous pressure drop associated with the wetting films. DiCarlo (2006) used a quasi-dynamic network model to study the saturation overshoot behind infiltration fronts. The model included the viscous pressure drop of the wetting phase during imbibition. Piri and Karpyn (2007) created a pore network representation of fracture void space from the fracture aperture map obtained through X-ray microtomography. They used a quasi-dynamic network model to simulate two-phase flow in a single fracture (Karpyn and Piri, 2007). Joekar-Niasar et al. (2010) studied the qualitative behavior of non-equilibrium capillary pressure theory (Stauffer, 1978; Hassanizadeh and Gray, 1990) using a dynamic network model. Tørå et al. (2012) extended the model developed by Aker et al. (1998) by incorporating the dynamics of the wetting layers using an approach similar to Singh and Mohanty (2003). They used this model to study saturation profiles during imbibition and the resistivity index at different capillary numbers. Sheng and Thompson (2013) extended the dynamic pore network model developed by Thompson (2002), and coupled it with a continuum-scale reservoir simulator. The coupling was performed by embedding pore networks inside a few 
gridblocks of the reservoir simulator.

Based on the literature review presented above, it is clear that while there have been numerous dynamic network modeling studies in the past, there is still a need for a physically-based dynamic network model capable of modeling pore-scale displacements in random networks and up-scaling them to the core scale. The previously-developed dynamic network models that include the complex pore-scale physics (Al-Gharbi and Blunt, 2005; Mogensen and Stenby, 1998; Dahle and Celia, 1999) lack the sufficient computational performance and can model two-phase flow in networks of few hundred to few thousand pores, and the ones that are capable of simulating flow in larger pore networks (Lee et al., 1995; Kamath et al., 1996; Xu et al., 1999) lack the necessary pore-scale physics such as the wetting-phase corner flow and snap-off displacements to quantitatively predict the multiphase flow properties.

We introduce an entirely new dynamic network model to perform physicallybased up-scaling of displacement processes from pore to core scale. The model takes into account viscous, capillary, and gravity forces as well as wettability effects. It incorporates all relevant displacement mechanisms and allows for variations in the order by which they take place under different flow regimes in response to changes in the capillary and Bond numbers. The wetting-phase corner flow with variable corner interface location responsive to changes in local capillary pressure is included in the model. Simultaneous injection of wetting and non-wetting fluids with constant flow rates from the inlet has been incorporated in the model, and it has enabled us to study the steady-state two-phase flow processes. Constant pressure boundary condition 
is used at the outlet. This platform is developed to run on massively parallel computer clusters and to bridge the gap between pore-scale displacements and core-scale processes.

In order to validate the model presented here, we performed three twophase miniature core-flooding experiments. The experiments were performed in a state-of-the-art core-flooding apparatus integrated with a high-resolution micro-CT imaging system. For the first time in pore-scale modeling literature, the in-situ contact angles measured from high-resolution micro-CT images of the pore space during experiments were used to design contact angle distributions in the digital pore network. Using the dynamic model, simulations were performed with identical flow rates and fluid properties as the experiments. The dynamic model was then rigorously validated by comparing the local saturation profiles, relative permeabilities, and fractional flow curves obtained from simulations against their experimental counterparts. The validated model was then used to study different dynamic processes that occur in, for instance, many enhanced oil recovery schemes.

In this paper, first, we give a detailed description of the dynamic model and techniques used to model two-phase flow at the core scale. Then, the miniature core-flooding experiments that are performed for the validation of the model are described in detail. This is followed by a rigorous validation of the simulation results against their experimental counterparts. The validated model is then used to study low-IFT and high viscosity flow processes. The dynamic effects observed in these high capillary number simulations are discussed in detail. Finally, we include a set of conclusions and final remarks. 


\section{Model Description}

\subsection{Network Representation of the Porous Medium}

We use three-dimensional random networks that are generated from highresolution micro-CT images of the rock samples used in the experiments presented here (see Dong and Blunt (2009) for more details on the network generation method). Two different pore networks representing the pore space in Bentheimer and Berea rock samples were used in this work. A $17 \mathrm{~mm}$ long section at the middle of the Berea core sample was scanned at a resolution of $2.49 \mu \mathrm{m}$. A pore network with a length of $16.3 \mathrm{~mm}$ and a square cross section of $3.11 \times 3.11 \mathrm{~mm}^{2}$ was generated. Twenty replicates of the $16.3 \mathrm{~mm}$ long Berea network were connected to build a larger network. Average properties from the original network (e.g., coordination number and throat radii) were used at the connection sites. A cylindrical pore network with a length of 76.4 $\mathrm{mm}$ and a diameter of $4.58 \mathrm{~mm}$ was cut from the larger network. We refer to this network as the Berea network hereinafter. The Bentheimer network was built using a similar approach.

The dimensions and petrophysical properties of the Bentheimer and Berea networks are listed in Tables 3 and 4, respectively. The pores and throats of our networks have rectangular, scalene triangular, and circular cross sections. Figure 1(a) shows a volume rendered gray scale image of the Berea sandstone core sample. This gray scale image is segmented using the histogram thresholding technique producing a pore-grain separated labeled image seen in Figure 1(b), where red and gray colors represent the pore and grain voxels, respectively. The gray-scale and pore-grain separated images are used to construct a pore network representative of the Berea core sample, as shown 
in Figure 1(c). In the pore network image, pores and throats are shown with red spheres and blue cylinders, respectively. Figure 1(d) shows a magnified image of a small section of the Berea pore network.

\subsection{Model Assumptions}

In this model, fluids are assumed to be Newtonian, incompressible, and immiscible. Fluid-fluid interfaces are assumed to be sharp with no diffusion taking place between two phases. Fluid flow inside pores and throats is described by Stokes or creeping flow. The clay inside the pore-space is assumed to be fully saturated with immobile water.

\subsection{Pore-Scale Displacements}

A series of pore-scale fluid displacements are carried out in the pore network to simulate two-phase flow displacements in porous media. Three fundamental types of pore-scale displacement mechanisms included in this dynamic model are piston-like, pore-body filling (cooperative filling), and snap-off.

The simulations start with fully water saturated pores and throats where all possible displacements from the inlet have been added to the system. Displacements occur in the order of highest-to-lowest displacement potential (see section 2.4) that takes into account the effects of capillary, viscous, and gravitational pressure drops. After each displacement, new displacements are added to the system based on the access of fluid phases to each other.

\subsection{Displacement Pressure Drop and Sequence}

The multiphase flow behavior in porous media is determined by the interlinked effects of capillary, viscous, and gravity forces. Therefore it is essential 
to include the effects of these three basic forces in the model. In this work, this is achieved by combining the capillary, viscous, and gravitational pressure drops of a displacement into a new parameter called the displacement pressure drop as given by equation (1).

$$
\begin{gathered}
\Delta P_{\text {disp }}=\Delta P_{\text {cap }}+\Delta P_{\text {visc }}+\Delta P_{\text {grav }} \\
N_{c}=\frac{\mu \nu}{\sigma} \\
B o=\frac{\Delta \rho g L^{2}}{\sigma}
\end{gathered}
$$

The contribution of each term in the displacement pressure drop to its total value depends on the capillary and Bond numbers (equations (2) and (3)). In the cases where the capillary and Bond numbers are both very low, capillary pressure dominates the flow behavior in porous media. However by increasing the capillary and Bond numbers the contributions of viscous and gravitational pressure drops, respectively, become more significant.

For piston-like displacements we use the Mayer-Stowe-Princen (MSP) method to calculate the capillary pressure drop of displacements (Mayer and Stowe, 1965; Princen, 1969a,b, 1970; Øren et al., 1998; Piri and Blunt, 2005a). In this method, an energy balance equation is written for the fluid configuration changes associated with a pore-scale displacement. By assuming the equilibrium conditions at constant temperature and combining the resultant equation with the Young-Laplace equation, the capillary pressure drop during a fluid configuration change is obtained. 


$$
\Delta P_{\text {visc }}=\left(P_{\text {inlet }}^{\text {invd }}-P_{\text {elm }}^{\text {invd }}\right)+\left(P_{\text {elm }}^{\text {def }}-P_{\text {outlet }}^{\text {def }}\right)
$$

The viscous pressure drop occurs due to the friction between layers of fluid that move with different velocities. We calculate the viscous pressure drop for both the invading and defending fluid phases. For the invading fluid phase the viscous pressure drop is calculated from the inlet of the pore network to the displacement location, and for the defending fluid phase, it is calculated from the displacement location to the outlet of the pore network as written in equation (4).

$$
\Delta P_{\text {grav }}=\left(\rho^{\text {invd }}-\rho^{\text {def }}\right) g h_{\mathrm{elm}}
$$

The gravitational pressure drop is the result of the hydrostatic pressure difference of the invading and defending fluid phases as given by equation (5), where $\rho^{\text {invd }}$ and $\rho^{\text {def }}$ are the densities of the invading and defending phases, respectively, $g$ is the acceleration of gravity, and $h_{\mathrm{elm}}$ is the height of the pore or throat involved in the displacement measured from the injection face of the medium.

$$
\Phi_{\text {disp }}=P_{\text {inlet }}^{\text {invd }}-P_{\text {outlet }}^{\text {def }}-\Delta P_{\text {disp }}
$$

Whether pore-scale displacements can take place and their sequence are determined by a parameter called the displacement potential. The displacement potential is defined as the difference of the driving force and the displacement pressure drop. The driving force is defined as the difference of the inlet pressure of the invading phase and the outlet pressure of the defending phase. The displacement potential for any given displacement is calculated 
using equation (6). Displacements can take place only if they have a positive potential, and they take place in the order of highest-to-lowest displacement potential.

\subsection{Volume Balance and Pressure Calculations}

Fluid phase pressure field calculation is regarded as the backbone of this dynamic model. The pressure field is obtained by writing volume balance equations for center and corner phases at each pore and solving the resulting system of linear equations with the appropriate boundary conditions.

$$
\sum_{j=1}^{n} q_{i j}=0
$$

Equation (7) gives the volume balance for pore $i$ where $q_{i j}$ is the flow rate between pores $i$ and $j$, and $n$ is the coordination number of pore $i$. For a pore that contains the non-wetting phase at its center and the wetting phase at its corners, this equation is written twice, once for the center phase and another time for the corner phase.

There are six possible configurations of two fluid phases in an assembly of two pores and a throat as shown in Figure 2. Pores and throats are shown by circles and cylinders for illustrative purposes only. They can have angular cross sections and contain center and corner phase locations. In configurations 1 and 4, no Main Terminal Meniscus (MTM) exists between pores $i$ and $j$, therefore the single phase flow equations are written for the center and corner phases of these configurations.

$$
q_{i j}^{p}=g_{i j}^{p}\left(P_{i}^{p}-P_{j}^{p}\right)
$$




$$
\frac{1}{g_{i j}^{p}}=\frac{1}{g_{\text {pore }, i}^{p}}+\frac{1}{g_{\text {throat }, i j}^{p}}+\frac{1}{g_{\text {pore }, j}^{p}}
$$

Equation 8 gives the corner flow and single-phase center flow, where $g_{i j}^{p}$ is the equivalent conductance of fluid phase $p$ between pores $i$ and $j$, and $P_{i}^{p}$ and $P_{j}^{p}$ are pressures of fluid phase $p$ in pores $i$ and $j$, respectively. $g_{i j}^{p}$ is calculated through equation 9.

$$
\begin{aligned}
& P_{c}^{\text {config. } 2}=P_{i}^{o}-P_{j}^{w} \\
& \Phi_{\text {config. } 2}^{\text {dra }}=P_{c}^{\text {config. } 2}-P_{c, i j}^{\text {dra }} \\
& \Phi_{\text {config. } 2}^{\text {imb }}=P_{c, i}^{\text {imb }}-P_{c}^{\text {config. } 2} \\
& q_{i j}=\left\{\begin{array}{lllll}
g_{i j}^{c e}\left(P_{i}^{n w}-P_{j}^{w}-P_{c, i j}^{\text {dra }}\right) & \text { if } & \Phi_{\text {config. } 2}^{\text {dra }}>\Phi_{\text {config. } 2}^{\text {imb }} & \text { and } & \Phi_{\text {config. } 2}^{\text {dra }}>0 \\
g_{i j}^{c e}\left(P_{i}^{n w}-P_{j}^{w}-P_{c, i}^{\text {imb }}\right) & \text { if } & \Phi_{\text {config. } 2}^{\text {imb }}>\Phi_{\text {config. } 2}^{\text {dra }} & \text { and } & \Phi_{\text {config. } 2}^{\text {imb }}>0 \\
0 & \text { if } & \Phi_{\text {config. } 2}^{\text {dra }}<0 & \text { and } & \Phi_{\text {config. } 2}^{\text {imb }}<0
\end{array}\right.
\end{aligned}
$$

In configurations where one or two MTM's exist between two pores (see Figure 2), two-phase flow equations are used to describe the center flow between the pores. The center flow equations for configuration 2 will be discussed here. In configuration 2, one oil-water MTM is located at the entrance of throat $i j$. The MTM can either move into throat $i j$ and cause a drainage-type flow, or retreat into pore $i$ and cause an imbibition-type flow depending on the local capillary pressure and threshold capillary pressures of pore $i$ and throat $i j$. Local capillary pressure for configuration 2 is written as 
the difference of oil pressure in pore $i$ and water pressure in pore $j$ (equation (10)). The potentials for two possible types of the MTM movement in configuration 2 are given by equations (11) and (12), where $P_{c, i j}^{\text {dra }}$ is the drainage threshold capillary pressure of throat $i j$ and $P_{c, i}^{\mathrm{imb}}$ is the imbibition threshold capillary pressure of pore $i$. As written in equation (13), if the drainage potential of the MTM is positive and greater than its imbibition potential, then a drainage-type flow will be considered for the MTM. However, if the imbibition potential is positive and greater than the drainage potential, then an imbibition-type flow will be considered for the MTM. In cases where both potentials are negative there will be no two-phase flow taking place between pores $i$ and $j$.

$$
\frac{1}{g_{i j}^{c e}}=\frac{1}{g_{\text {pore }, i}^{\text {nw }}}+\frac{1}{g_{\text {throat }, i j}^{w}}+\frac{1}{g_{\text {pore }, j}^{w}}
$$

The equivalent center flow conductance, $g_{i j}^{c e}$, in configuration 2 , is calculated through equation 14. The center flow conductances are calculated by using the equations proposed by Øren et al. (1998) and Patzek and Silin (2001), while the corner flow conductances are calculated by equations proposed by Hui and Blunt (2000).

The resultant system of linear equations is solved by using the the MUMPS package, which is a massively parallel sparse matrix solver (Amestoy et al., 2001, 2006).

\subsection{Boundary Conditions}

In order to replicate the experimental conditions during simulations and to be able to study steady-state two-phase flow processes, wetting and nonwetting fluids are injected simultaneously at constant flow rates from the inlet 
of the medium. This is similar to the work by Hashemi et al. $(1998,1999 b, a)$ in which both the wetting and non-wetting fluids act as the invader and the defender. In our model, drainage and imbibition processes are simulated by changing the inlet flow rates. The direction in which the inlet flow rates change determines the type of two-phase flow process we simulate.

$$
\sum_{j=1}^{n} q_{i j}^{p}=q_{i n l e t}^{p}
$$

Material balance is written for the inlet as shown in equation (15) where $q_{\text {inlet }}^{p}$ is the flow rate of phase $p$ at the inlet. Pressures of wetting and nonwetting phases at the inlet are obtained by solving the material balance equations.

At the outlet of the medium, constant pressure boundary conditions are enforced. Since the local capillary pressure at the pore scale cannot be zero, a non-zero capillary pressure is enforced at the outlet. The outlet capillary pressure has the value below which no network-spanning cluster of the non-wetting phase can exist in the system. During drainage, when the nonwetting phase reaches the outlet of the network, the pressures of the wetting and non-wetting fluids are averaged in a partition of the network adjacent to the outlet, and capillary pressure is determined in that partition. This capillary pressure is set as the outlet capillary pressure. However, if the capillary number is high, the outlet capillary pressure can be overestimated using this method. Therefore, the capillary pressure set at the outlet is reduced gradually in small increments to find the value below which the connection of the non-wetting phase to the outlet is lost completely. This critical value is set as the outlet capillary pressure. This procedure is performed automatically 
by the model as soon as the breakthrough of the non-wetting phase occurs.

The outlet capillary pressure is kept constant during the simulations. One should note that the values set as the outlet capillary pressure are insignificant compared to the macroscopic pressure drops along the network.

\subsection{Corner Interface Handling}

The location of Arc Menisci (AM's) determine the area open to corner and center flows and have a significant impact on pressure values and thereby relative permeabilities as well as other predicted properties. This dynamic model allows the location of AM's to be updated based on the local capillary pressure and receding and advancing contact angles. This is similar to the approach that is often used in quasi-static network models in determining threshold capillary pressure for a snap-off displacement.

$$
P_{c}=\sigma_{o b}\left(\frac{1}{r_{1}}+\frac{1}{r_{2}}\right)
$$

When a new AM is formed in a pore element, its location is determined by calculating its distance from the apex of the corner in which it is formed. The Young-Laplace equation (equation (16)) combined with a geometrical relationship is used to determine the apex-meniscus distance. After an AM is formed, the local capillary pressure might change due to changes in fluid phase pressure distribution. The moving contact angle $\left(\theta_{\text {mov }}\right)$ is defined as the contact angle with which a fluid-fluid interface moves and it has a constant value during the interface movement. When the local capillary pressure in a pore element changes, an AM in the pore element will move only if its contact angle is equal to $\theta_{\text {mov }}$, otherwise the AM will hinge and its contact 
angle will change by keeping a fixed apex-meniscus distance. As an example, as shown in Figure 3(a), if $P_{c}$ increases and the contact angle is equal to the receding contact angle, then the AM will move toward the corner apex with a fixed contact angle. In Figure 3(b), on the other hand, $P_{c}$ decreases, and the contact angle is lower than the advancing contact angle, therefore the AM will hinge away from the corner apex with a fixed apex-meniscus distance.

\subsection{Parallelization}

The computational challenges in dynamic network models have limited the size of the mediums that were studied with previously-developed models (see Tables 1 and 2). They have also hindered studies of heterogeneous mediums at the pore scale. Calculating the fluid phase pressure field and updating the fluid-fluid interface locations and displacement potentials at each time step, and updating the continuity flags of neighboring fluid phase locations after each displacement are computationally the most demanding steps in this dynamic model. We take advantage of greater memory and computational resources available on massive computer clusters. Data parallelism through three-dimensional domain decomposition was employed in designing the parallel algorithms.

In Figure 4, a domain decomposition scheme is illustrated, in which the domain of the problem is divided into, in this case, 27 subdomains and each subdomain is assigned to one processor. In this work, the pore network is divided into multiple blocks based on the availability of the computational resources, and each block is assigned to one processor. The pores and throats whose center coordinates lie inside the boundaries of a block are considered as local elements to that block and their information is stored on the memory 
of the processor assigned to that block. The computations related to a pore or throat are done by the processor to which the element is local. Consider two neighboring blocks $\mathrm{A}$ and $\mathrm{B}$ as shown in Figure 5. Pore $i$ and throat $i j$ are local to block A, which is assigned to processor a, and pore $j$ is local to block B, which is assigned to processor b. Processor a is responsible for computations related to pore $i$ and throat $i j$, and processor $\mathrm{b}$ is responsible for computations related to pore $j$. However, during some computations, processors a and $\mathrm{b}$ might also need the information related to pore $j$ and throat $i j$, respectively. Therefore pore $j$ and throat $i j$ are considered as interblock elements to blocks A and B, respectively, and part of their information needs to be stored in the memory of their respective processors as well. The information related to inter-block elements such as fluid phase occupancy and pressures needs to be communicated during simulations between neighboring processors. MPI (Message Passing Interface) (Gabriel et al., 2004) libraries are used to carry out communications between processors in this model.

\subsection{The Model Algorithm}

The general outline of the model is explained in this section. Algorithm flow chart of the model is presented in Figure 6. The first step is reading the simulation parameters and the network data and decomposing it among the available processors. Then, the initial fluid phase is assigned to the pores and throats and the isolated elements are detected in the pore network. A parallel clustering algorithm is developed for the cluster detection in a decomposed domain. Next, the absolute permeability and porosity of the pore network are calculated. By examining the elements connected to the inlet of the network, all possible displacements from the inlet are detected and added to 
the displacement lists.

Now that the model has been initialized, the first flow-rate step is started by setting the first pair of wetting and non-wetting phase flow rates as the inlet boundary condition. The pressure field is computed with the new inlet flow rates. Using an iterative procedure the pressure calculation, corner interface update, area-volume calculations, and phase conductance calculations are repeated until the pressure values converge. The available volume of each phase during a time step is calculated and the most favorable displacement is detected. If the available volume is sufficient to carry out the most favorable displacement, the displacement takes place. Otherwise the simulation goes to the next time step. After each displacement, new pore-scale displacements are detected and added to the system. The displacements continue to take place until no displacement with a positive potential is left. At that point, the system has reached the steady-state condition. The relative permeabilities, phase saturations, pressure distributions, and fluid occupancy data are reported at steady-state points. The simulation ends if it is at the final flow-rate step. Otherwise it goes to the next flow-rate step.

\section{Experiments}

To validate our dynamic pore network model, we conducted three sets of two-phase miniature core-flooding experiments in Betheimer and Berea sandstone core samples. The experiments were performed using a stateof-the-art core-flooding apparatus integrated with a high-resolution X-ray micro-computed tomography imaging system. In this section, the experimental procedures and conditions, fluid and rock properties, flow rates, image 
segmentation and analysis techniques, in-situ contact angle measurements, and pressure drop analysis method are described. The experimental setup used in this work is described in Alizadeh et al. (2014) and Khishvand et al. (2014) as well.

\subsection{Experimental Procedures and Conditions}

Table 5 lists the experiment numbers along with the core samples, experimental procedure and conditions, and fluids used in this study.

Dimensions and petrophysical properties of the core samples are listed in Table 6. Figure 7 shows the Berea sandstone core sample used in this work along with its pore network representation. An automated helium porosimeter-permeameter that employs a pressure decay technique was used to measure porosity and absolute permeability of a large Bentheimer core sample from which we cut the miniature samples for the experiments. Porosity was also obtained from high-resolution micro-CT images of the core samples. Porosity values obtained from the helium porosimeter-permeameter and high-resolution micro-CT images were in agreement. Brine permeability was measured for the Berea sandstone core sample.

Properties of the fluids used in the experiments are listed in Table 7. Viscosity measurements were performed at the experimental temperature and pressure conditions for each fluid using an electromagnetic viscometer. Densities of the fluids were measured at the experimental temperature and ambient pressure conditions using a DMA 5000 density meter that utilizes the oscillating U-tube method. Densities were not expected to change significantly at the experimental pressures of 0.90 and $2.76 \mathrm{MPa}$. IFT values were measured at the experimental temperature and pressure conditions us- 
ing a high pressure pendent drop technique (see Saraji et al. (2013) for more details on the experimental method).

Each experiment was started with a fully brine saturated core sample $(\mathrm{Sw}=1)$, that was vertically placed in the core-flooding apparatus. The sample was held inside a custom-built micro core holder made of carbon fiber. It was kept under confining pressure. All the wetted parts of the core holder and the core-flooding system were made of Hastelloy or other corrosion resistant material. The fluids were injected from the bottom of the core. Experiments 1 and 2 were performed using the steady-state method meaning that both the wetting and non-wetting fluids were injected during each test.In these experiments, the drainage tests were performed by gradually reducing the brine flow rate and increasing the oil flow rate. The imbibition tests were started after the drainage tests by increasing the brine flow rate and decreasing the oil flow rate. Tables 8 and 9 list the brine and oil flow rates and their corresponding capillary numbers in Experiments 1 and 2, respectively. In Experiment 3, the drainage test was performed with the last oil and brine flow rates that were used during drainage in Experiment 2. The imbibition test in Experiment 3 was started with the same initial brine saturation as in Experiment 2. The phase saturations and pressure drops were verified to be the same at the end of drainage in Experiments 2 and 3. The imbibition test in Experiment 3 was performed by shutting down the oil pump and increasing the water flow rate to $0.1 \mathrm{~cm}^{3} / \mathrm{min}$. Experiment 3 was performed to investigate the effects of high capillary number imbibition on the residual oil saturation. Oil and brine flow rates and their corresponding capillary numbers in Experiment 3 are listed in Table 10. 
In Experiment 1, the full length and cross-sectional area of the Bentheimer core sample was scanned at a resolution of $2.6 \mu \mathrm{m}$ in all directions at specified steady-state points in Table 8. The Berea core sample in Experiments 2 and 3 was too long to be scanned at full length at every steady-state point. Therefore, certain locations along the Berea core were selected as scan spots. The location of these spots and their lengths and numbers are listed in Table 11. The Berea core in Experiments 2 and 3 was scanned at a resolution of $2.49 \mu \mathrm{m}$, in all directions, at fractional flow steps and scan spots specified in Tables 9 and 10.

\subsection{Steady-State Criteria for Experiments}

During the experiments, after the brine and oil flow rates were set to their predetermined values, the pressure drop along the core sample was monitored carefully. Four differential pressure transducers with various ranges were used to measure the pressure drop along the cores. When the pressure drop reached a stable value, the lower resolution scanning of the core sample was started. The core sample was scanned repeatedly until the saturation difference between two consecutive scans was less than 1\%. This would ensure the establishment of the steady-state condition. At that point, the core sample was scanned with a higher resolution and the pressure drop data were recorded for relative permeability calculations.

\subsection{Micro-CT Image Segmentation and Analysis}

The micro-CT images of the core samples were analyzed using the Avizo ${ }^{\circledR}$

Fire software. A technique similar to the one used by Alizadeh et al. (2014) and Khishvand et al. (2014) was used for the analysis of the micro-CT images. 
The dry core samples were scanned to obtain reference images before the flow experiments were started (see Figure 8(a)). First, the non-local means filter was applied to the reference images to reduce the noise. The reference images were then segmented using histogram thresholding technique as shown in Figure 8 (b).

The analysis of the wet images obtained during the flow experiments (see Figure 8(c)) was started, similar to dry images, by applying the non-local means filter. Then, the wet images were registered with the dry images and were multiplied by the binary pore-grain separated images to separate the pore space and grains. The resulting images were then segmented for the separation of the oil and brine phases (see Figure 8(d)). Arithmetic operations were performed on these labeled images to obtain average saturations and saturation profiles along the core sample.

\subsection{In-Situ Contact Angle Measurements}

The micro-CT images obtained during the two-phase flow experiments were used to measure the contact angles between the wetting and non-wetting phases in the pore space. This is similar to the technique used by Andrew et al. (2014). First a corner of a pore containing the brine and oil phases was selected. Then the point where the brine-oil interface line intersects the solid surface was detected. At that point, two lines one tangent to the solid surface and the other one tangent to the brine-oil interface were drawn. The angle between these two lines through the brine phase gave the brine-oil contact angle at that corner. Figure 9 shows in-situ contact angle measurements in pore corners in the Berea sandstone core during the experiments. Brine, oil, and grains are depicted by colors blue, red, and gray, respectively. The green 
lines show the tangents to the rock surface and the brine-oil interface at the triple contact points.

Table 12 lists the average, minimum, and maximum brine-oil contact angles measured at multiple points in the Bentheimer and Berea sandstone core samples during drainage and imbibition processes of Experiments 1, 2, and 3. These values were used in identifying the receding and advancing brineoil contact angle distributions in the Bentheimer and Berea pore networks in the simulations presented here. The average, minimum, and maximum values of brine-oil contact angle distributions in the Bentheimer and Berea pore networks are also listed in Table 12 for comparison.

\subsection{Fluid Phase Potential Difference}

In the experiments, the core sample was placed vertically and the fluids were injected from the bottom of the core. Therefore, the effect of gravity needed to be included in the potential difference in the Darcy's equation. For this purpose, when the core sample was fully saturated with brine, the injection pumps were shut down and the pressure transducers were reset to zero. As a result, the value shown by the pressure transducers was the potential difference for the brine phase and it needed to be corrected for the oil phase relative permeability calculations.

$$
\Delta \Phi_{o}=\Delta \Phi_{b}+\left(\rho_{b}-\rho_{o}\right) g L
$$

Equation (17) was used to convert the pressure difference value read from transducers to the oil phase potential difference. In this equation, $\Delta \Phi_{o}$ and $\Delta \Phi_{b}$ are potential differences of oil and brine phases respectively, $\rho_{b}$ and $\rho_{o}$ 
are brine and oil densities, respectively, $g$ is the acceleration of gravity, and $L$ is the length of the core sample. After obtaining the potential differences for each phase, the relative permeabilities were calculated using the Darcy's equation.

\section{Validation}

In this section, first simulation procedures and conditions, steady-state criteria for simulations, and contact angle distributions used in simulations are described. This is followed by the comparison of simulation and experimental results.

\subsection{Simulation Procedures and Conditions}

The dynamic model was used to perform simulations in pore networks constructed from high-resolution micro-computed tomography images of the core samples in which the experiments were performed. The properties of the pore networks representative of the Bentheimer and Berea core samples used in the experiments are presented in Tables 3 and 4, respectively. The properties of the fluids that were measured during the experiments and presented in Table 7 were used in the simulations. The simulations were performed using the same brine and oil flow rates used during the experiments. These flow rates and their corresponding capillary numbers are presented in Tables 8, 9, and 10. For each experiment listed in Table 5, there is an equivalent simulation with the same number.

The simulations were started with fully brine saturated pore networks.

The vertical orientation of the core sample was simulated by using the ap- 
propriate coordinates of pore elements as the height in gravity pressure drop calculations (see equation (5)).

\subsection{Steady-State Criteria for Simulations}

In the simulations, the pore-scale displacements were carried out in the order of highest-to-lowest displacement potential (see section 2.4). The displacements were carried out until no displacement with a positive potential was left in the system. At that point, the system had reached the steady-state condition for one set of inlet flow rates. The simulation and experimental results are compared against each other at steady-state points.

\subsection{Contact Angles Used in Simulations}

In-situ measurements of the brine-oil contact angles during the experiments provided us with realistic estimations of contact angle distributions in the real rock pore space (see section 3.4). The average, minimum, and maximum values of the random distributions of the receding and advancing contact angles used in the Berea and Bentheimer pore networks are listed in Table 12. One should note that the contact angles measured during drainage are receding. However, the contact angles measured during imbibition are either hinging or advancing and accordingly their average values are between receding and advancing contact angles. Therefore, the advancing contact angle values used in the simulations are larger than the contact angles measured during imbibition.

\subsection{Comparison with Experimental Data}

Simulations 1, 2, and 3 were performed as the equivalents of Experiments 1,2 , and 3, respectively. Simulation 1 that was performed in the Bentheimer 
sandstone network took about 15 hours to complete on 64 processors with $2000 \mathrm{MHz}$ speed. While Simulations 2 and 3 that were performed in the Berea sandstone network took about 2 weeks to complete on 200 processors with $2000 \mathrm{MHz}$ speed. The experimental and simulation data presented here relate to steady-state conditions that were established at each fractional flow point. We compare each simulation with the corresponding experiment through local saturation points, fractional flow curves, and relative permeabilities.

\subsubsection{Simulation 1}

In Experiment 1, the full cross section and length of the Bentheimer core sample was scanned at selected fractional flow numbers listed in Table 8 after the steady-state condition was achieved. The scanning resolution was $2.6 \mu \mathrm{m}$ in all directions. After eliminating the micro-CT images close to the inlet and outlet of the core sample that were corrupted due to geometric penumbra and high noise, 4000 two-dimensional slices along the core sample were obtained for each fractional flow point. These slices were then processed and segmented. For brine saturation profile measurements, these 4000 slices were divided into 40 groups of 100 slices. The thickness of each group of slices was $0.26 \mathrm{~mm}$. The volume of solid, oil, and brine phases were determined in each group of slices and subsequently the brine saturation in each group was calculated. The distance of the middle of each slice group from the inlet was used to plot the measured brine saturation versus position along the core sample. In Simulation 1, the pore network was divided into 50 partitions along the length of the network. The thickness of each partition was 0.26 $\mathrm{mm}$. The brine saturation was calculated at each fractional flow point after achieving the steady-state condition. Figure 10 shows the saturation profiles 
obtained from Experiment 1 and Simulation 1 at selected fractional flows.

The saturation profiles from Simulation 1 show encouraging agreement with their experimental counterparts.

In Figure 15, fractional flows of brine versus average brine saturations obtained from Experiment 1 and Simulation 1 are presented at each steady-state point. Fractional flow curves obtained from Simulation 1 and Experiment 1 agree well.

Due to the failure of the pressure transducers during Experiment 1, the pressure drop data obtained in this experiment were not useable. Here, we compare the relative permeability data obtained from Simulation 1 against the data available in the literature for the same rock sample. Oil-brine twophase relative permeability data on Bentheimer sandstone presented by Alizadeh and Piri (2014) and Øren et al. (1998) were used here for comparison. Øren et al. (1998) used a paraffinic oil and a synthetic brine $(3 \% \mathrm{NaCl})$, while Alizadeh and Piri (2014) used Soltrol 170 and a synthetic brine (2 wt $\% \mathrm{CaCl}_{2}, 12$ wt $\% \mathrm{NaI}$, and $0.01 \mathrm{wt} \% \mathrm{NaN}_{3}$ ) as the non-wetting and wetting phases, respectively. Øren et al. (1998) reported the experiments at ambient pressure and temperature, while Alizadeh and Piri (2014) performed their experiments at $5.52 \mathrm{MPa}$ and ambient temperature. They present drainage and imbibition relative permeabilities versus brine saturation. Figure 12 shows the relative permeabilities obtained from Simulation 1 and the literature data on drainage and imbibition. As it is apparent from this figure, the relative permeabilities from Simulation 1 are in good agreement with the experimental values from the literature. The predicted residual oil saturation also matches the experimental values. 
4.4.2. Simulation 2

In Experiment 2, the middle section of the Berea core sample was scanned in all fractional flows. The bottom and top parts of the core were scanned in selected fractional flows (see Table 9). The scanning resolution was 2.49 $\mu \mathrm{m}$ in all directions.

The brine saturation profiles in the bottom, middle, and top sections of the medium at the end of first drainage and last imbibition fractional flows obtained from Experiment 2 and Simulation 2 are presented in Figure 13. The experimental data points in this figure are obtained by dividing the $2 \mathrm{D}$ micro-CT slices into 60 groups of 30 slices. The thickness of each group was $74.7 \mu \mathrm{m}$. The simulation data are obtained by dividing the pore network into 60 partitions in the same middle, bottom, and top sections of the medium as Experiment 2, and calculating the brine saturation in each partitions.

Figure 14 shows saturation profiles in full length of the medium obtained from Simulation 2. Experiment 2 results are also depicted in this figure in locations where the core sample was scanned. Each data point in this figure represents the saturation in a $0.747 \mathrm{~mm}$ long section of the medium.

As seen in Figures 13 and 14, the saturation profiles from Simulation 2 compare well with those from Experiment 2. Since this experiment was performed under capillary-dominated displacement regime, the saturation profiles are uniform along the length of the sample, a condition that is critical for steady-state relative permeability measurements (Alizadeh and Piri, 2014).

Fractional flows of brine versus saturation obtained from Experiment 2 and Simulation 2 at each steady-state point are presented in Fig 15. The frac- 
tional flow curves show good agreement between the measured and predicted data.

Relative permeabilities from Simulation 2 are compared against relative permeabilites from Experiment 2 and the data available in the literature. The experimental oil-brine two-phase relative permeability data on Berea sandstone presented by Oak (1990) are used for comparison purposes. Oak (1990) performed the two-phase experiments with brine $\left(5 \% \mathrm{NaCl}, 0.5 \% \mathrm{CaCl}_{2}\right)$ and dodecane at 5.52 MPa and ambient temperature conditions. Figure 16 shows the relative permeabilities from Simulation 2, Experiment 2, and Oak (1990). In this figure, brine saturation of Experiment 2 is obtained from the middle section of the core sample. As shown in this figure, Simulation 2 and Experiment 2 relative permeability and saturation values show good agreement. Simulation 2 and Experiment 2 data show a slight discrepancy with the data from Oak (1990), which may be attributed to the differences in the samples used. For instance, the absolute permeabilities of the samples used by Oak (1990) were $200 \mathrm{mD}, 800 \mathrm{mD}$, and $1000 \mathrm{mD}$ while our sample had an absolute permeability of $623 \mathrm{mD}$. Furthermore, the core samples used by Oak (1990) were fired before performing the flow experiments. It is noteworthy that in the quasi-static pore network models that have been used to match the data from Oak (1990) the pore networks have higher porosity and clay content than the Berea sandstone network used in this work (see, for instance, Lerdahl et al. (2000); Piri and Blunt (2005a,b)).

\subsubsection{Simulation 3}

In Experiment 3, the middle, bottom, and top sections of the Berea core sample were scanned at a resolution of $2.49 \mu \mathrm{m}$ (in all directions) at all imbi- 
bition fractional flows. The drainage part of this experiment was performed

to establish the same initial brine saturation as in Experiment 2. This was confirmed by a lower resolution scan at the end of drainage. The middle, top, and bottom section micro-CT images obtained at the end of imbibition fractional flows were analyzed and grouped using the same approach as Experiment 2. Figure 17 shows saturation profiles in full length of the medium obtained from Simulation 3 and Experiment 3. Each data point in this figure represents the saturation in a $0.747 \mathrm{~mm}$ long section of the medium. Simulation 3 saturation profiles are in good agreement with their experimental counterparts. Due to the dynamic effects caused by the higher capillary number during imbibition (see Table 10), in Experiment 3 and Simulation 3, there is a slight difference between brine saturation in the bottom, middle, and top sections of the medium. The predicted residual oil saturation agrees closely with the experimental value.

\section{Simulation of Different Processes}

Having rigorously validated the dynamic model against the experimental data, in this section, we use it to study other processes. Three simulations were conducted in the Bentheimer sandstone network introduced earlier. All three simulations were performed with the brine and oil flow rates used in Simulation 1 (see Table 8). However, different IFT and viscosity values were used in each simulation. Following the simulation numbering used in the previous section, simulations in this section are numbered 4, 5, and 6. Properties of the fluids and ranges of the capillary number in Simulations 4, 5, and 6 are listed in Table 13. The contact angle distributions used in Simulation 
1 (see Table 12) were used in Simulations 4, 5, and 6. The simulation results in this section are compared against the results from Simulation 1 with the purpose of investigating the effects of low IFT and high viscosity of wetting phase on relative permeabilities, residual oil saturation, and fractional flow curves.

\subsection{Simulation 4-Surfactant Flooding}

Surfactant flooding is an enhanced oil recovery technique that is used to increase recovery by reducing IFT between displacing phase and oil. By lowering IFT the capillary forces that are responsible for trapping oil in the pore scale are reduced and the effect of viscous forces becomes significant (Lake, 1989; Green and Willhite, 1998). Simulation 4 was performed in order to investigate how a low IFT value might affect relative permeabilities and residual oil saturation. The IFT value in this simulation was $1.0 \mathrm{mN} / \mathrm{m}$ as opposed to $46.34 \mathrm{mN} / \mathrm{m}$ in Simulation 1 (see Tables 7 and 13). Due to the reduced IFT value, the capillary numbers in Simulation 4 are higher than those in Simulation 1 and they range between $3 \times 10^{-6}$ and $1 \times 10^{-3}$ (see Tables 8 and 13).

As seen in Figure 18, relative permeabilities of wetting and non-wetting phases in Simulation 4 are higher than those in Simulation 1 for a given saturation. Furthermore, a wider range of saturation is observed during drainage and imbibition in Simulation 4. Residual oil saturation in this simulation is $21.36 \%$, which is $15.75 \%$ lower than that in Simulation 1 with a higher IFT.

In Simulation 4, due to the low IFT value used, the capillary pressure drop associated with pore-scale displacements becomes lower and the viscous 
pressure drop becomes a more significant term in the displacement pressure drop (see section 2.4). Therefore, pore-scale displacements take place in paths that result in lower viscous pressure drop. These flow paths can conduct the same flow rate with lower viscous pressure drop. In other words, there is less resistance in these flow paths. For this reason, higher relative permeabilities are obtained in Simulation 4 for a given saturation.

In this simulation, the rise in the capillary number makes the effect of viscous pressure drop more dominant. Therefore, the pore-scale displacements that are closer to the displacement front become more favorable. Consequently, piston-like displacements close to the displacement front are favored over snap-off displacements ahead of the front. This change in the favored displacement type results in less trapping of the non-wetting phase. Therefore, in this simulation residual oil saturation is lower than that in Simulation 1.

\subsection{Simulation 5 - Polymer Flooding}

Polymer flooding is an enhanced oil recovery technique in which polymer is added to the displacing phase in order to decrease its mobility and increase the sweep efficiency of the flooding process. The added polymer decreases the mobility of the displacing phase by increasing its viscosity, and, in some cases, by decreasing its permeability. It is generally believed that adding polymer by itself does not decrease the residual oil saturation if the capillary number is kept constant (Lake, 1989). However, in this section, we compare two cases with the same flow rates but different viscosities and, therefore, different capillary numbers. The effects that are seen in this simulation are the result of an increase in the capillary number. In this simulation, the 
viscosity ratio of the wetting to non-wetting phase was 5.0 as opposed to 1.31 in Simulation 1 (see Tables 7 and 13). Due to the increase in viscosity of the wetting phase, the capillary numbers during imbibition in Simulation 5 are higher than those in Simulation 1 and they range between $8 \times 10^{-7}$ and $8 \times 10^{-5}$ (see Tables 8 and 13 ).

In Figure 19(a) drainage relative permeabilities obtained from Simulations 5 and 1 are compared. Since the capillary number during drainage is almost the same in these two simulations, drainage relative permeabilities do not show a significant difference. However, drainage steady-state points in Simulation 5 are established at lower wetting-phase saturations than those in Simulation 1 for a given fractional flow. This can be attributed to the fact that the higher viscosity of the wetting phase in Simulation 5 increases the viscous pressure drop of the wetting phase and makes it more difficult for oil to displace the wetting phase with the same flow rates as in Simulation 1.

As seen in 19(b), wetting-phase relative permeability does not show a big difference in Simulations 5 and 1. Oil relative permeability, however, is greater in Simulation 5 for a given saturation. Furthermore, the residual oil saturation in Simulation 5 is $24.10 \%$, which is $13.01 \%$ lower than residual oil saturation in Simulation 1. Because of the higher wetting-phase-to-oil viscosity ratio in Simulation 5, the flow pattern becomes more stable during imbibition (Lenormand et al., 1988), meaning that the wetting-phase-to-oil displacements closer to the inlet are favored. Therefore, piston-like displacements closer to the inlet are favored over snap-off displacements ahead of the displacement front. The change in the flow pattern results in less trapping of oil. Therefore, oil relative permeability is higher and residual oil saturation 
is lower in Simulation 5.

\subsection{Simulation 6 - Surfactant-Polymer Flooding}

Polymers are sometimes used in surfactant flooding in order to increase the sweep efficiency of the process by lowering the mobility of the displacing phase (Lake, 1989). Simulation 6 was performed to study the combined effect of low IFT and high viscosity of the wetting phase on relative permeabilities and residual oil saturation. Due to the low IFT value and high viscosity of the wetting-phase, the capillary numbers in Simulation 6 are higher than those in Simulations 1, 4, and 5 and they range between $4 \times 10^{-6}$ and $4 \times 10^{-3}$ (see Tables 8 and 13).

As shown in Figure 20(a), the dynamic effects observed during drainage in Simulation 4 are present and more significant in Simulation 6. Saturation ranges in Simulations 4 and 6 are almost identical during drainage. However, steady-state points are established at different saturations in these simulations for a given fractional flow. Wetting-phase relative permeability is higher in Simulation 6 for a given saturation. Oil relative permeability is higher in early stages and then it becomes lower in late drainage.

Because in Simulation 6 viscosity ratio of wetting phase to oil is 5.0, during drainage, oil has an unstable front (Lenormand et al., 1988). The low IFT value and the high viscosity of the wetting phase in this simulation makes the wetting-phase viscous pressure drop the dominant force in displacements. Therefore, oil-to-wetting-phase displacements that are closer to the outlet are preferred. In other words, displacements tend to take place in the paths that cause the least wetting-phase viscous pressure drop. However, these displacement paths do not necessarily have the least resistance to oil. 
Furthermore, there may be some un-swept banks of water that would cause more viscous pressure drop in oil flow. Additionally, the high viscosity of the wetting phase increases the wetting-phase pressure in the corners and keeps the wetting layers thick in Simulation 6. Because of the above-mentioned reasons, drainage wetting-phase relative permeability is higher in Simulation 6 than its counterpart in Simulation 4; and drainage oil relative permeability becomes lower especially during late stages in Simulation 6 .

In imbibition, the dynamic effects that caused an increase in relative permeabilities in Simulation 4 are seen in Simulation 6, as well (see Figure 20(b)). Wetting-phase relative permeabilities from Simulations 4 and 6 are almost identical. Oil relative permeabilities from Simulation 6, however, are a little higher than their counterparts from Simulation 4. Residual oil saturation in Simulation 6 is $17.27 \%$, which is $4.09 \%$ and $19.84 \%$ lower than residual oil saturations in Simulations 1 and 4, respectively.

In Simulation 6, since the wetting-phase-to-oil viscosity ratio is 5.0, the displacement front during imbibition is stable (Lenormand et al., 1988). The dominant force in displacements is the viscous pressure drop of the wetting phase. Therefore, there is less trapping of oil in this simulation compared to Simulation 4 in which wetting-phase-to-oil viscosity ratio is 1.31. This shows that adding a polymer to a surfactant solution can increase the oil recovery by reducing the mobility of the displacing phase and consequently changing the flow pattern and inhibiting the snap-off displacements.

Fractional flow curves of wetting-phase obtained from Simulations 1, 4, 5, and 6 during drainage and imbibition are shown in Figure 21. As seen in Figure 21(a), the lowest wetting-phase saturation during drainage is obtained 
in Simulations 4 and 6, in which the IFT value is $1.0 \mathrm{mN} / \mathrm{m}$. In imbibition, as seen in Figure 21(b), the lowest residual saturation is obtained from Simulation 6 , where the IFT value and wetting-phase-to-oil viscosity ratio are $1.0 \mathrm{mN} / \mathrm{m}$ and 5.0, respectively. Following Simulation 6, Simulations 4 and 5 have the second and third lowest residual oil saturations, respectively.

In order to evaluate the validity of the dynamic behavior observed in Simulations 4, 5, and 6, here, we briefly discuss the experimental observations reported in the literature for high capillary number flows. du Prey (1973) conducted two-phase flow experiments in artificial porous media in order to study the parameters affecting relative permeabilities and residual saturations. It was shown that higher capillary numbers result in higher relative permeabilities. Furthermore, the viscosity ratio was shown to affect relative permeabilities and the displacement pattern. Amaefule and Handy (1982) studied the effect of IFT on relative permeabilities using core-flooding tests in Berea samples. Oil and water relative permeabilities increased with decreasing IFT. The residual oil saturation was shown to decrease by lowering IFT at high capillary numbers. Chatzis and Morrow (1984) conducted coreflooding experiments in water-wet sandstones to investigate the displacement of initially continuous and residual oil with the increase in the capillary number. The decrease in residual saturation of initially continuous oil started at capillary numbers lower than $10^{-5}$. The rise in relative permeabilities and the decrease in residual oil saturation by increasing the capillary number, that are observed in Simulations 4, 5, and 6 of the present work, are consistent with the experimental results reported in the above-mentioned studies. Lenormand et al. (1988) studied two-phase flow in porous media in a wide range of 
capillary numbers and viscosity ratios using a dynamic pore network model and micromodel tests. Stable displacement, viscous fingering, and capillary fingering flow patterns were identified in the results from simulations and experiments. The higher viscosity ratio of the displacing to displaced phase at high capillary numbers was shown to produce a stable displacement front and reduce the trapping of non-wetting phase in imbibition. On the other hand, the lower viscosity ratio of the displacing to displaced phase at high capillary numbers was shown to generate viscous fingers of the displacing phase across the medium. Toussaint et al. (2012) discussed the flow patterns observed during drainage experiments in disordered porous media. The relative permeabilities and residual oil saturations obtained from Simulations 4,5 , and 6 , in the present work, reflect the displacement patterns that are in agreement with the results reported in the literature (Lenormand et al., 1988; Toussaint et al., 2012).

The effects of IFT, viscosity ratio, and capillary number on displacement pattern, relative permeabilities, and residual saturations that are observed in Simulations 4, 5, and 6 show some of the capabilities of our dynamic model. This model can also be used to study size effects and obtain quantitative scaling laws by running simulations on networks with different sizes and keeping all the other simulation parameters the same. Furthermore, the effects of micro-fractures, pore-space heterogeneities, and different pore types in carbonate rocks on two-phase flow properties can be studied using this model. Quasi-static models, which do not include viscous and gravitational forces, and majority of the previously-developed dynamic models, which run on small networks of a few hundred to a few thousand pores, cannot be used 
to study the above-mentioned phenomena.

\section{Conclusions}

A new dynamic pore network model that is capable of simulating flow at the core scale was developed. This dynamic pore network model can be used as a platform to study various two-phase flow processes in porous media. The dynamic model included six critical elements: (1) the pressure drops associated with capillary, viscous, and gravity forces, (2) wetting-phase corner flow, (3) corner interface adjustments based on changes in local capillary pressure, (4) simultaneous injection of wetting and non-wetting phases from the inlet of the medium at constant flow rates, (5) parallelization based on a threedimensional domain decomposition scheme that made simulations on large core-size samples possible, and (6) constant pressure boundary condition at the outlet. These features make the model, to the best of our knowledge, the first of its kind that can be used to bridge the gap between pore and core scales for a wide range of flow problems in different conventional rock types. For the validation of the dynamic model, three two-phase miniature flow experiments were performed using a state-of-the-art micro core-flooding apparatus that is integrated with a high-resolution micro-CT scanner. A Bentheimer sandstone core with the length of $12.98 \mathrm{~mm}$ and the diameter of 4.97 $\mathrm{mm}$, and a Berea sandstone core with the length of $76.40 \mathrm{~mm}$ and the diameter of $4.58 \mathrm{~mm}$ were used in the experiments. The dynamic model was used to perform simulations with the identical medium size, flow rates, and fluid properties as the experiments in the pore networks that were constructed from high-resolution micro-CT images of the core samples. The micro-CT 
images obtained from the two-phase flow experiments were used to obtain insitu brine-oil contact angles during various stages of the experiments. These measurements were then used to design realistic contact angle distributions in the pore networks for the simulations.

The predicted saturation profiles, fractional flow curves, relative permeabilities, and residual oil saturations compared successfully against their experimental counterparts. Furthermore, the relative permeabilities and residual oil saturations from simulations and experiments were consistent with the data available in the literature. The encouraging agreement between the measured results and the predicted results is the manifestation of the relevant two-phase flow physics along with the realistic boundary conditions and fluid and rock properties used in the model. This makes the pore network model introduced in this work a powerful platform for studying complex dynamic two-phase flow processes in porous media in core-size rock samples. Moreover, this dynamic model can be used for coupling with continuum-scale reservoir simulators

The validated model was used to simulate low-IFT and high-viscosity two-phase flow processes that occur in, for instance, many enhance oil recovery schemes. Three simulations with different IFT values and viscosity ratios were performed to study the effects of high capillary number on relative permeabilities and residual oil saturation. The relative permeabilities increased with the rise of the capillary number. The residual oil saturation decreased as the flow pattern became more viscous dominated. The lowest residual oil saturation was obtained in the case where the viscosity ratio of the wetting phase to oil was the highest and the IFT value was the lowest. The 
dynamic effects observed during the high capillary number simulations were in agreement with the results reported from microfluidics and core-flooding tests available in the literature.

\section{Acknowledgments}

This work was supported by DOE Financial Assistance Agreement DEFE0004832. We also acknowledge the financial support of Hess Corporation and the School of Energy Resources at the University of Wyoming. We extend our gratitude to Hu Dong and Sven Roth of iRock Technologies for generating the pore networks for our samples. We also thank Mahdi Khishvand, Henry Plancher, Morteza Akbarabadi, and Soheil Saraji of Piri Research Group at the University of Wyoming for their help with the experiments and laboratory measurements. All the data presented in this work can be obtained from Dr. Mohammad Piri of the University of Wyoming (mpiri@uwyo.edu).

\section{References}

Aghaei, A., 2014. Direct pore-to-core up-scaling of displacement processes: Experimentation and dynamic pore network modeling. PhD dissertation, University of Wyoming, Laramie, WY, USA.

Aker, E., Maløy, K. J., Hansen, A., Batrouni, G. G., 1998. A two-dimensional network simulator for two-phase flow in porous media. Transport in Porous Media 32(2), 163-186. 
Al-Gharbi, M. S., Blunt, M. J., 2005. Dynamic modeling of two-phase drainage in porous media. Physical Review E 71(016308), 016308-1016308-16.

Alizadeh, A. H., Khishvand, M., Ioannidis, M. A., Piri, M., 2014. Multi-scale experimental study of carbonated water injection: An effective process for mobilization and recovery of trapped oil. Fuel 132, 21917235.

Alizadeh, A. H., Piri, M., 2014. The effect of saturation history on threephase relative permeability:An experimental study. Water Resources Research 50 .

Amaefule, J. O., Handy, L. L., 1982. The effect of interfacial tensions on relative oil/water permeabilities of consolidated porous media. SPE Journal $22,371-381$.

Amestoy, P. R., Guermouche, A., L'Excellent, J.-Y., Pralet, S., 2006. Hybrid scheduling for the parallel solution of linear systems. Parallel Computing 32(2), 136-156.

Amestoy, P. R., I. S. Duff, J. K., L'Excellent, J.-Y., 2001. A fully asynchronous multifrontal solver using distributed dynamic scheduling. SIAM Journal of Matrix Analysis and Applications 23(1), 15-41.

Andrew, M., Bijeljic, B., Blunt, M. J., 2014. Pore-scale contact angle measurements at reservoir conditions using x-ray microtomography. Advances in Water Resources 68, 24-31.

Blunt, M. J., Jackson, M. D., Piri, M., Valvatne, P. H., 2002. Detailed 
physics, predictive capabilities and macroscopic consequences for porenetwork models of multiphase flow. Advances in Water Resources 25(8-12), 1069-1089.

Blunt, M. J., King, P., 1991. Relative permeabilities from two- and threedimensional pore-scale network modelling. Transport in Porous Media 6(4), 407-433.

Chatzis, I., Morrow, N. R., 1984. Correlation of capillary number relationships for sandstone. SPE Journal 24.

Constantinides, G. N., Payatakes, A. C., 2000. Effects of precursor wetting films in immiscible displacement through porous media. Transport in Porous Media 38(3), 291-317.

Dahle, H. K., Celia, M. A., 1999. A dynamic network model for two-phase immiscible flow. Computational Geosciences 3(1), 1-22.

DiCarlo, D. A., 2004. Experimental measurements of saturation overshoot on infiltration. Water Resources Research 40.

DiCarlo, D. A., 2006. Quantitative network model predictions of saturation behind infiltration fronts and comparison with experiments. Water Resources Research 42.

Dong, H., Blunt, M. J., 2009. Pore-network extraction from microcomputerized-tomography images. Physcial Review E 80, 036307.

du Prey, E. J. L., 1973. Factors affecting liquid-liquid relative permeabilities of a consolidated porous medium. SPE Journal 13, 39-47. 
Dullien, F. A. L., 1992. Porous Media: Fluid Transport and Pore Structure, 2nd Edition. Academic Press, San Diego.

Fatt, I., 1956a. The network model of porous media I. Capillary pressure characteristics. Transactions of the American Institute of Mining, Metallurgical, and Petroleum Engineers 207, 144-159.

Fatt, I., 1956b. The network model of porous media II. Dynamic properties of a single size tube network. Transactions of the American Institute of Mining, Metallurgical, and Petroleum Engineers 207, 160-163.

Fatt, I., 1956c. The network model of porous media III. Dynamic properties of networks with tube radius distribution. Transactions of the American Institute of Mining, Metallurgical, and Petroleum Engineers 207, 164-181.

Fourie, W., Said, R., Young, P., Barnes, D., 2007. The simulation of pore scale fluid flow with real world geometries obtained from X-ray computed tomography. Proceedings of the COMSOL Conference, Boston.

Gabriel, E., Fagg, G. E., Bosilca, G., Angskun, T., Dongarra, J. J., Squyres, J. M., Sahay, V., Kambadur, P., Barrett, B., Lumsdaine, A., Castain, R. H., Daniel, D. J., Graham, R. L., Woodall, T. S., September 2004. Open MPI: Goals, concept, and design of a next generation MPI implementation. In: Proceedings, 11th European PVM/MPI Users' Group Meeting. Budapest, Hungary, pp. 97-104.

Gingold, R., Monaghan, J., 1997. Smoothed particle hydrodynamics: theory and application to non-spherical stars. Monthly Notices of the Royal Astronomical Society 181, 37517389. 
Green, D. W., Willhite, G. P., 1998. Enhanced Oil Recovery, 1st Edition. SPE, Richardson, TX.

Hashemi, M., Dabir, B., Sahimi, M., 1999a. Dynamics of two-phase flow in porous media: Simultaneous invasion of two fluids. AIChE Journal 45, 1365.

Hashemi, M., Sahimi, M., Dabir, B., 1998. Percolation with two invaders and two defenders: Volatile clusters, oscillations, and scaling. Physical Review Letters 80, 3248.

Hashemi, M., Sahimi, M., Dabir, B., 1999b. Monte Carlo simulation of twophase flow in porous media: Invasion with two invaders and two defenders. Physica A 267, 1.

Hassanizadeh, S. M., Gray, W. G., 1990. Mechanics and thermodynamics of multiphase flow in porous media including interphase boundaries. Advances in Water Resources 13, 169-186.

Hughes, R. G., Blunt, M. J., 2000. Pore scale modeling of rate effects in imbibition. Transport in Porous Media 40(3), 295-322.

Hui, M. H., Blunt, M. J., 2000. Effects of wettability on three-phase flow in porous media. Journal of Physical Chemistry B 104(16), 3833-3845.

Inamuro, T., Ogata, T., Tajima, S., Konishi, N., 2004. A Lattice Boltzmann method for incompressible two-phase flows with large density differences. Journal of Computational Physics 198, 628644. 
Joekar-Niasar, V., Hassanizadeh, S. M., 2012. Analysis of fundamentals of two-phase flow in porous media using dynamic pore-network models: A review. Critical Reviews in Environmental Science and Technology 42:18, 1895-1976.

Joekar-Niasar, V., Hassanizadeh, S. M., Dahle, H. K., 2010. Non-equilibrium effects in capillarity and interfacial area in two-phase flow: Dynamic porenetwork modelling. Journal of Fluid Mechanics 655.

Kamath, J., Xu, B., Lee, S., Yortsos, Y., 1996. Pore network modeling of laboratory experiments on heterogeneous carbonates. Paper SPE 36681, Proceedings of the SPE Annual Technical Conference and Exhibition, Denver, Colorado, 6-9 October.

Karpyn, Z. T., Piri, M., 2007. Prediction of fluid occupancy in fractures using network modeling and X-ray microtomography. I: Data conditioning and model description. Physical Review E 76(016315), doi: 10.1103/PhysRevE.76.016315.

Khishvand, M., Akbarabadi, M., Piri, M., 2014. A pore-level investigation of residual trapping, dynamic effects, and matrix dissolution in sandstone and carbonate using x-ray microtomography. submitted to Microporous and Mesoporous Materials.

Knudsen, H. A., Hansen, A., 2002. Relation between pressure and fractional flow in two-phase flow in porous media. Physical Review E 65(5).

Koplik, J., Lasseter, T., 1985. Two-phase flow in random network models of porous media. SPE Journal 25. 
Koshizuka, S., Tamako, H., Oka, Y., 1995. A particle method for incompressible viscous flow with fluid fragmentation. Computational Fluid Dynamics Journal 4(1), 291746.

Lake, L. W., 1989. Enhanced Oil Recovery, 1st Edition. Prentice Hall, Englewood Cliffs, NJ.

Lee, S. H., Padmanabhan, L., Al-Sunaidi, H. A., 1995. Simulation of linear displacement experiments on massively parallel computers. Paper SPE 30721, Proceedings of the SPE Annual Technical Conference and Exhibition, Dallas, TX, 22-25 October.

Lenormand, R., Touboul, E., Zarcone, C., 1988. Numerical models and experiments on immiscible displacements in porous media. Journal of Fluid Mechanics 189(April), 165-187.

Lerdahl, T. R., Øren, P. E., Bakke, S., 2000. A predictive network model for three-phase flow in porous media. Paper SPE 59311, Proceedings of the SPE/DOE Symposium in Improved Oil Recovery, Tulsa, Oklahoma, 3-5 April.

Li, H., Pan, C., Miller, C., 2005. Pore-scale investigation of viscous coupling effects for two-phase flow in porous media. Physical Review E 72, 026705.

Løvoll, G., Méheust, Y., Måløy, K. J., Aker, E., Schmittbuhl, J., 2005. Competition of gravity, capillary and viscous forces during drainage in a twodimensional porous medium, a pore scale study. Energy 30, 861-872. 
Mayer, R. P., Stowe, R. A., 1965. Mercury porosimetry-breakthrough pressure for penetration between packed spheres. Journal of Colloid Science 20, 893-911.

Mogensen, K., Stenby, E., 1998. A dynamic two-phase pore-scale model of imbibition. Transposrt in Porous Media 32.

Nguyen, V. H., Sheppard, A. P., Knackstedt, M. A., Pinczewski, W. V., 2006. The effect of displacement rate on imbibition relative permeability and residual saturation. Journal of Petroleum Science and Engineering 52.

Nordhaug, H. F., Celia, M., Dahle, H. K., 2003. A pore network model for calculation of interfacial velocities. Advances in Water Resources 26(10), 1061-1074.

Oak, M. J., 1990. Three-phase relative permeability of water-wet Berea. Paper SPE 20183, Proceedings of the SPE/DOE Seventh Symposium on Enhanced Oil Recovery, Tulsa, Oklahoma, 22-25 April.

Øren, P. E., Bakke, S., 2003. Reconstruction of berea sandstone and porescale modelling of wettability effects. Journal of Petroleum Science and Engineering 39, 177-199.

Øren, P. E., Bakke, S., Arntzen, O. J., 1998. Extending predictive capabilities to network models. SPE Journal 3(4), 324-336.

Ovaysi, S., Piri, M., 2010. Direct pore-level modeling of incompressible fluid flow in porous media. Journal of Computational Physics 229, 7456177476. 
Ovaysi, S., Piri, M., 2011. Pore-scale modeling of dispersion in disordered porous media. Journal of Contaminant Hydrology 124, 68-81.

Patzek, T. W., 2001. Verification of a complete pore network simulator of drainage and imbibition. SPE Journal 6(2), 144-156.

Patzek, T. W., Silin, D. B., 2001. Shape factor and hydraulic conductance in noncircular capillaries. I. One-phase creeping flow. Journal of Colloid and Interface Science 236(2), 295-304.

Piri, M., Blunt, M. J., 2005a. Three-dimensional mixed-wet random pore-scale network modeling of two- and three-phase flow in porous media. I. Model description. Physical Review E 71(026301), doi:10.1103/PhysRevE.71.026301.

Piri, M., Blunt, M. J., 2005b. Three-dimensional mixed-wet random porescale network modeling of two- and three-phase flow in porous media. II. Results. Physical Review E 71(026302), doi:10.1103/PhysRevE.71.026302.

Piri, M., Karpyn, Z. T., 2007. Prediction of fluid occupancy in fractures using network modeling and X-ray microtomography. II: Results. Physical Review E 76(016316), doi: 10.1103/PhysRevE.76.016316.

Premože, S., Tasdizen, T., Bigler, J., Lefohn, A., Whitaker, R., 2003. Particle-based simulation of fluids. Computer Graphics Forum 22, 3.

Princen, H. M., 1969a. Capillary phenomena in assemblies of parallel cylinders I. Capillary rise between two cylinders. Journal of Colloid and Interface Science 30(1), 69-75. 
Princen, H. M., 1969b. Capillary phenomena in assemblies of parallel cylinders II. Capillary rise in systems with more than two cylinders. Journal of Colloid and Interface Science 30(3), 359-371.

Princen, H. M., 1970. Capillary phenomena in assemblies of parallel cylinders III. Liquid columns between horizontal parallel cylinders. Journal of Colloid and Interface Science 34(2), 171-184.

Sahimi, M., 2010. Flow and Transport in Porous Media and Fractured Rock, 2nd Edition. Wiley-VCH, Los Angeles.

Saraji, S., Goual, L., Piri, M., 2013. Wettability of sc-CO 2 /water/Quartz systems: Simultaneous measurement of contact angle and interfacial tension at reservoir conditions. Langmuir 29, 6856-6866.

Schaefer, C. E., DiCarlo, D. A., Blunt, M. J., 2000. Experimental measurement of air-water interfacial area during gravity drainage and secondary imbibition in porous media. Water Resources Research 36(4), 885-890.

Sheng, Q., Thompson, K., 2013. Dynamic coupling of pore-scale and reservoir-scale models for multiphase flow. Water Resources Research 49.

Singh, M., Mohanty, K. K., 2003. Dynamic modeling of drainage through three-dimensional porous material. Chemical Engineering Science 58, 118.

Stauffer, F., 1978. Time dependence of the realtions between capillary pressure, water content and conductivity during drainage of porous media. IAHR Symposium on Scale Effects in Porous Media, Thessaloniki, Greece, 3.35-3.52. 
Tartakovsky, A., Meakin, P., 2005. A smoothed particle hydrodynamics for miscible flow in three-dimensional fractures and two-dimensional RayleighTaylor instability. Journal of Computational Physics 207, 61017624.

Thompson, K. E., 2002. Pore-scale modeling of fluid transport in disordered fibrous materials. AIChE Journal 48(7).

Tørå, G., Øren, P.-E., Hansen, A., 2012. A dynamic network model for twophase flow in porous media. Transport in Porous Media 92, 145-164.

Toussaint, R., Måløy, K. J., Méheust, Y., Løvoll, G., Jankov, M., Schäfer, G., Schmittbuhl, J., 2012. Two-phase flow: Structure, upscaling, and consequences for macroscopic transport properties. Vadose Zone Journal 11(3), doi=10.2136/vzj2011.0123.

Valvatne, P. H., Blunt, M. J., 2004. Predictive pore-scale modeling of twophase flow in mixed wet media. Water Resources Research 40, W07406.

van der Marck, S. C., Matsuura, T., Glas, J., 1997. Viscous and capillary pressures during drainage: Network simulations and experiments. Physical Review E 56(5), 5675-5687.

Xu, B., Kamath, J., Yortsos, Y., Lee, S., 1999. Use of pore-network models to simulate laboratory corefloods in a heterogeneous carbonate sample. SPE Journal 4(3), 179-186.

Zhu, Y., Fox, P. J., Morris, J. P., 1999. A pore-scale numerical model for flow through porous media. International Journal for Numerical and Analytical Methods in Geomechanics 23, 88117904. 

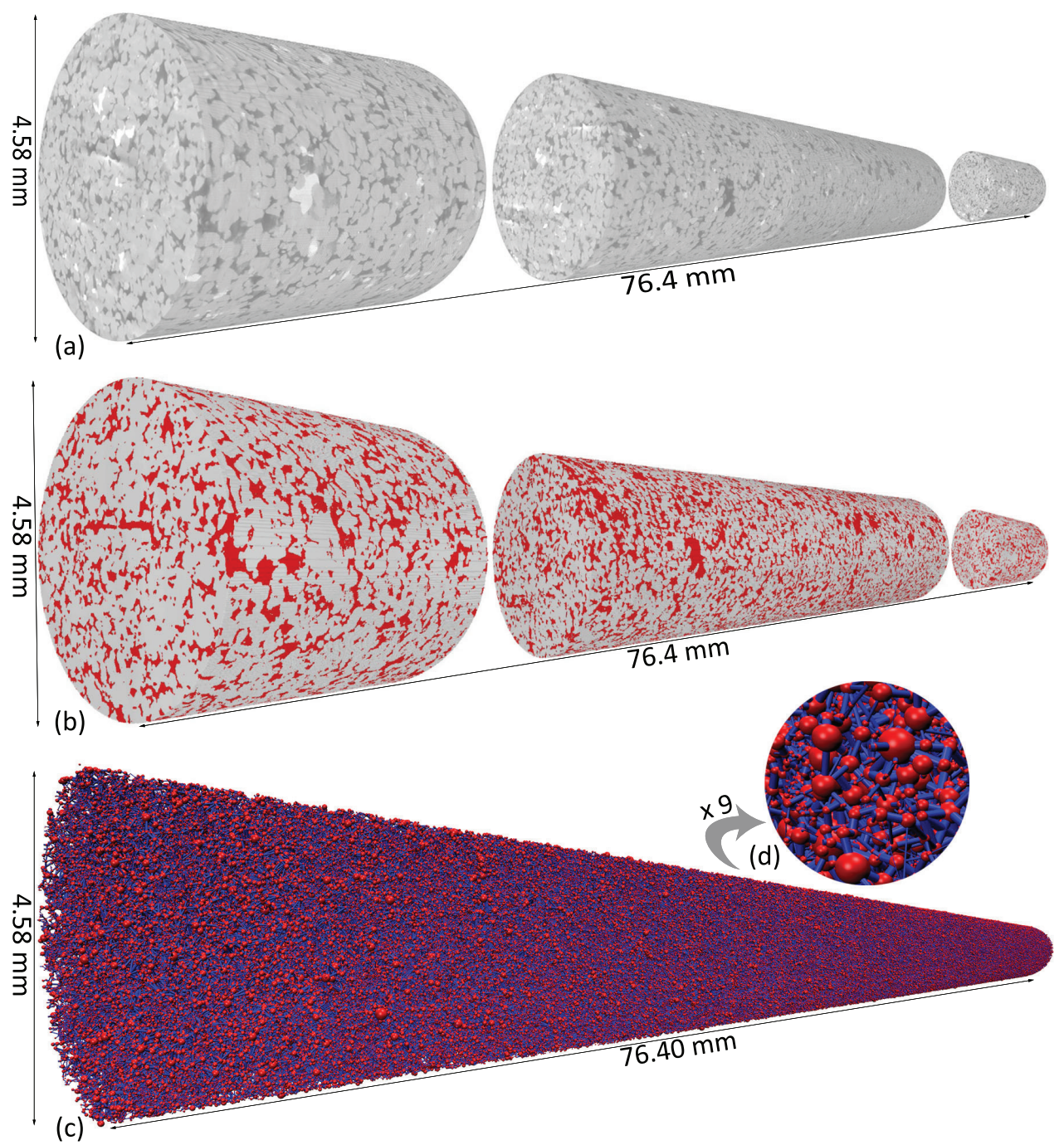

Figure 1: Pore network representation of the Berea sandstone core sample. (a) Volume rendered gray-scale images of the Berea core sample scanned with a micro-CT scanner at a resolution of $2.49 \mu \mathrm{m}$. (b) Volume rendered pore-grain separated images of the Berea core sample. Red and gray colors represent the pore and grain voxels, respectively. (c) Pore network generated from images (a) and (b). Red and blue colors represent the pores and throats in the network, respectively. For illustrative purposes pores and throats are shown with circular cross-section here. (d) A magnified image of a small section of the Berea pore network. 


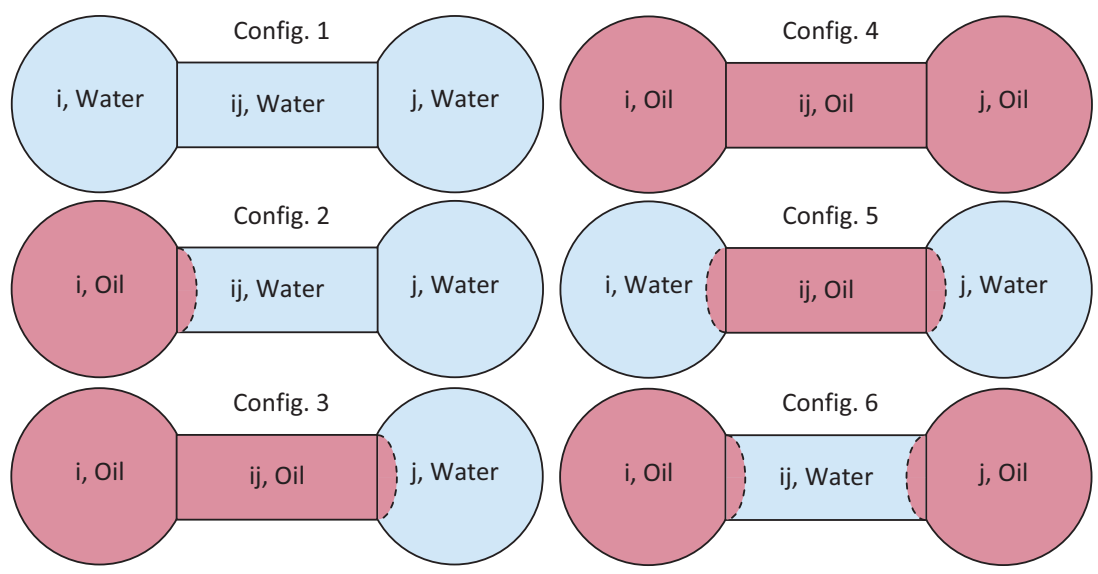

Figure 2: All possible fluid configurations in an assembly of two pores and a throat. Pores and throats are shown with circles and rectangles for illustrative purposes only. They can have angular cross-sections and contain a center as well as a corner phase. Only the center phases are illustrated here. The corners will contain water if the elements are angular. The dotted curvatures show the Main Terminal Menisci (MTM's) present between the wetting and non-wetting phases. 


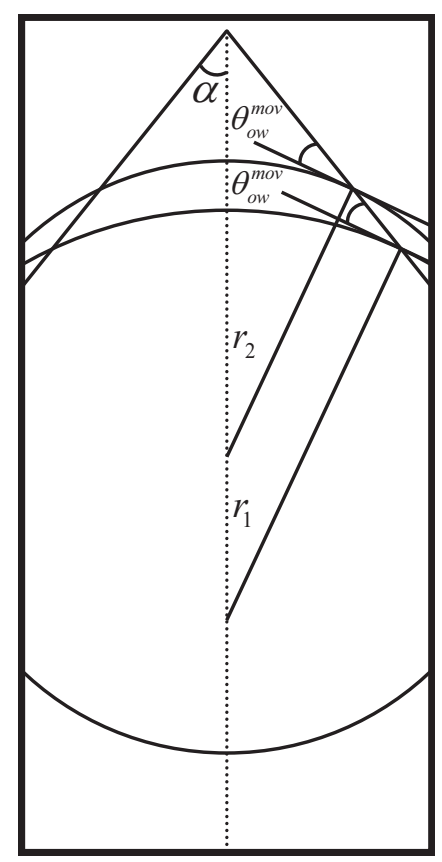

(a)

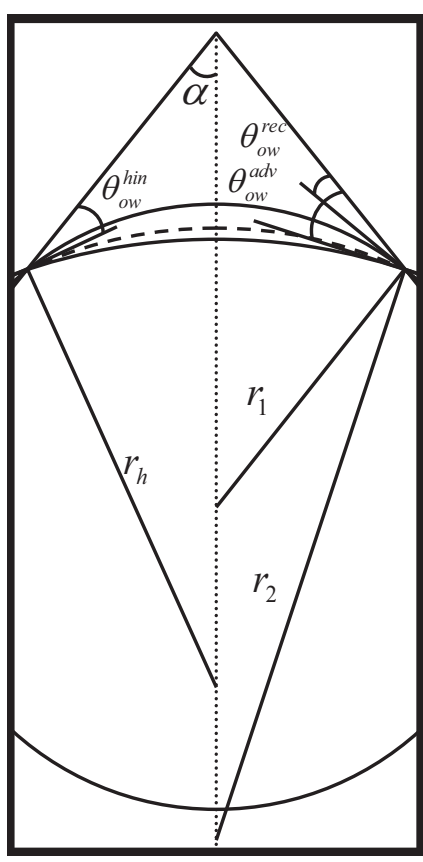

(b)

Figure 3: Corner interface adjustments based on changes in local capillary pressure. (a) The contact angle is equal to the receding contact angle. The corner interface, i.e., AM moves towards the apex of the corner as the local capillary pressure increases. (b) The contact angle is greater than the receding contact angle. The corner interface hinges as the local capillary pressure decreases. 

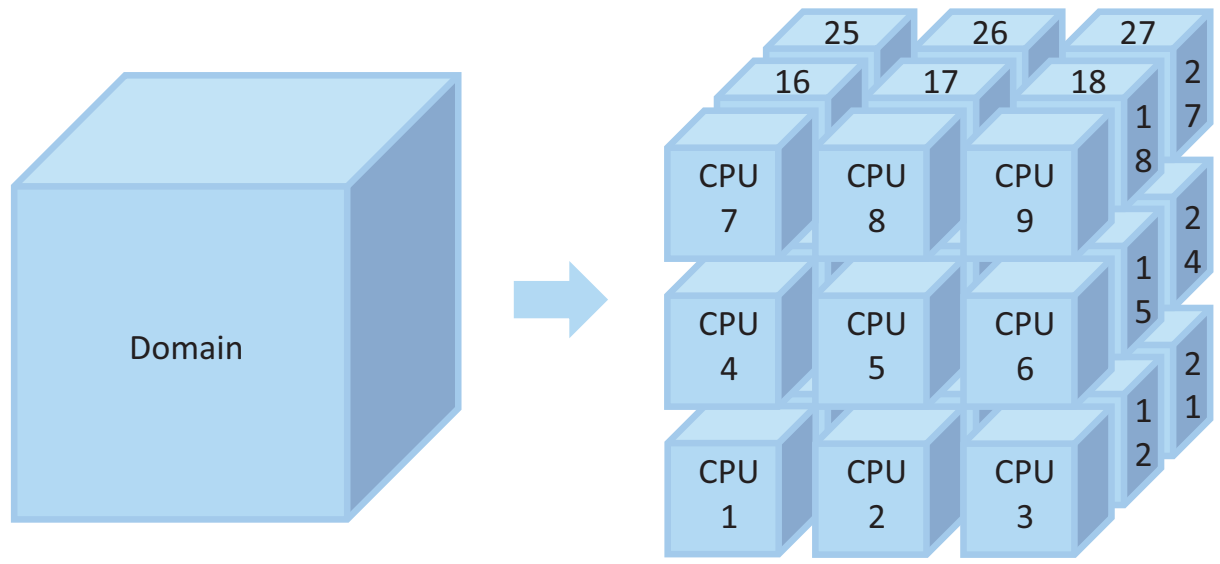

Figure 4: Domain decomposition. The domain of the problem, in this figure, is divided into 27 blocks and each block is assigned to a processor. 


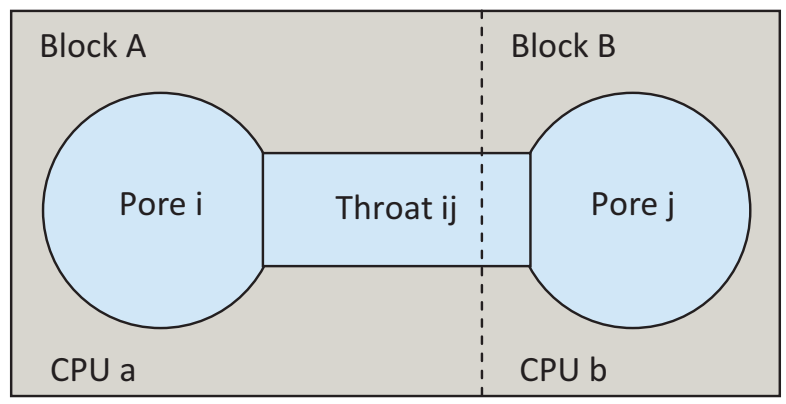

Figure 5: Local and inter-block elements. Pore i and throat ij are local to block A, which is assigned to processor a. Pore $\mathrm{j}$ is local to block $\mathrm{B}$, which is assigned to processor $\mathrm{b}$. Pore $\mathrm{j}$ and throat $\mathrm{ij}$ are inter-block elements to blocks A and B, respectively. 


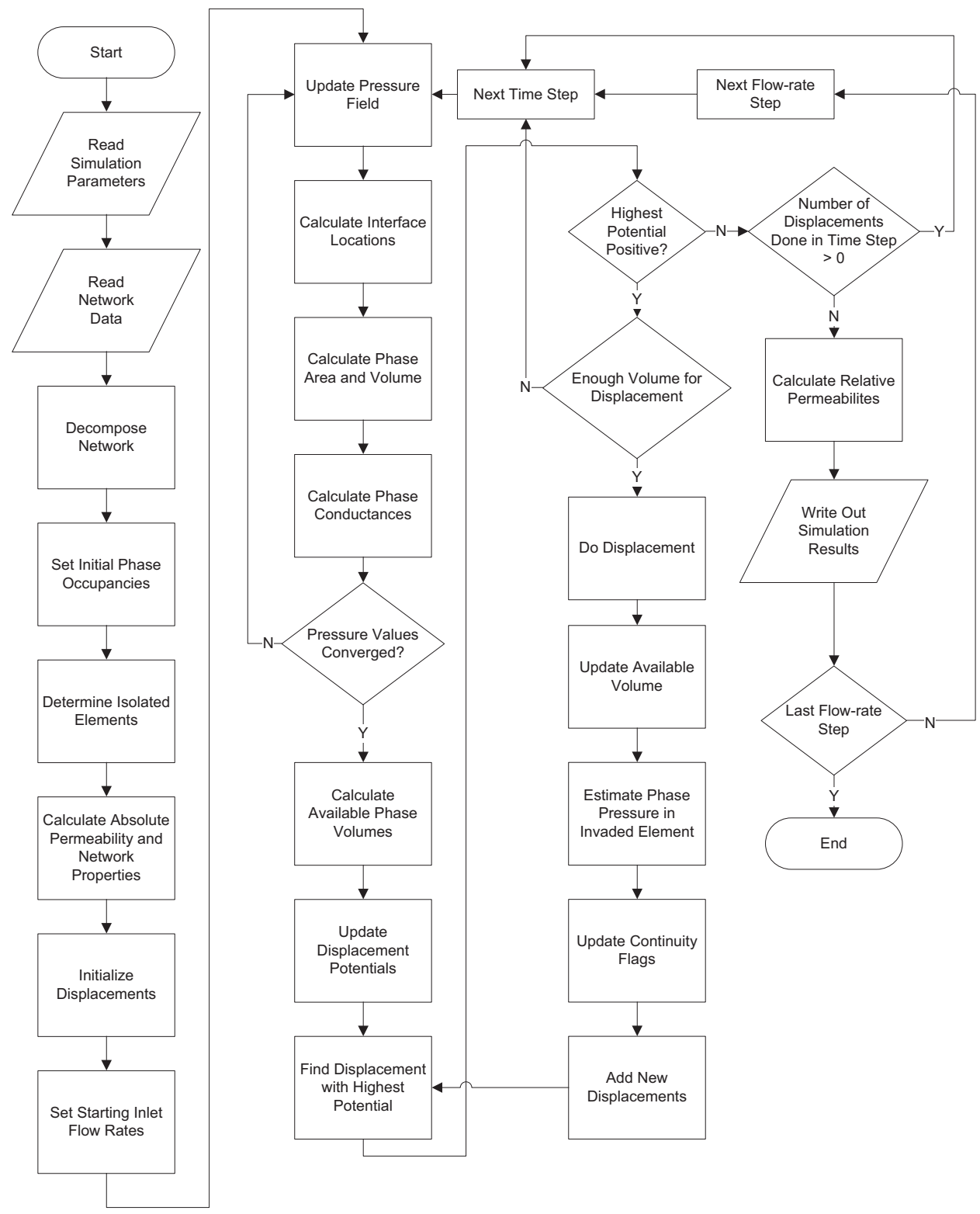

Figure 6: The algorithm flow chart of the dynamic pore network model presented in this work. 


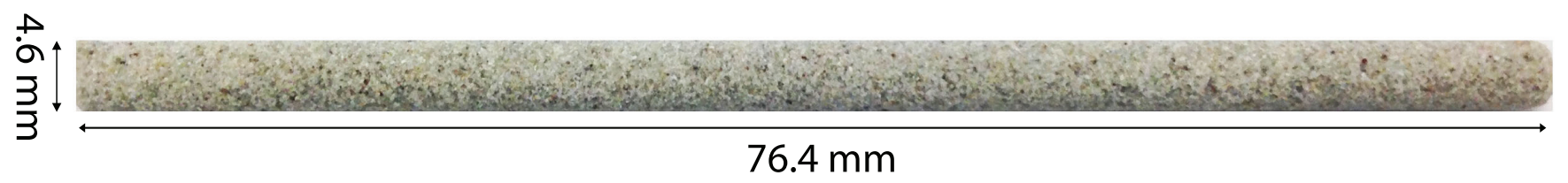

(a)

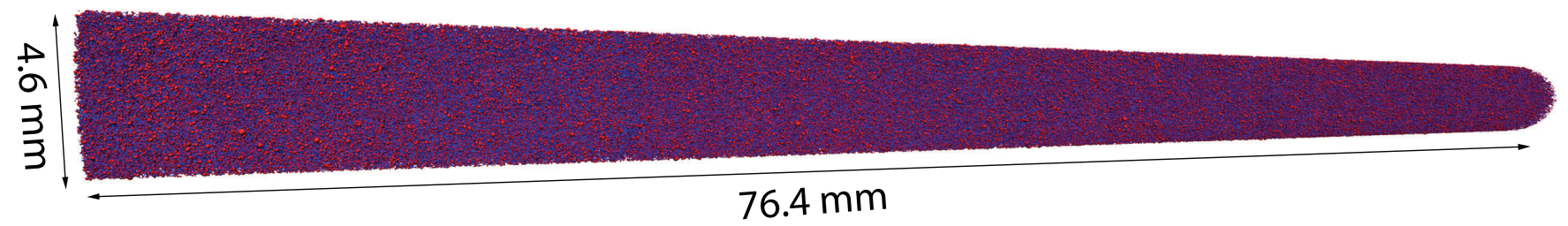

(b)

Figure 7: (a) The Berea sandstone core sample used in this work. (b) The pore network representation of the Berea sample used in this work. Pores and throats are depicted by red spheres and blue cylinders, respectively. 

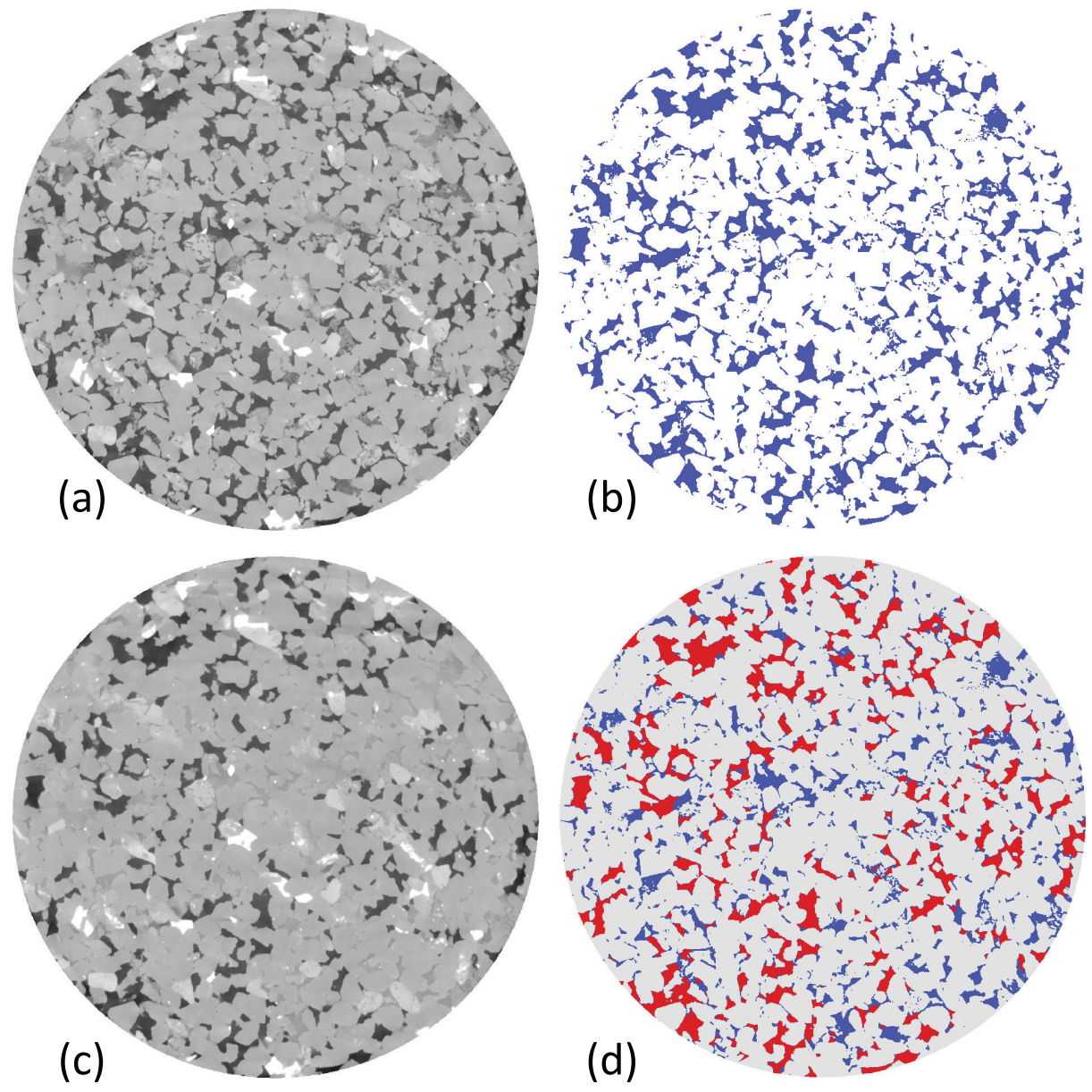

Figure 8: Three-phase segmentation of high resolution micro-CT images of Berea sandstone scanned at $2.49 \mu \mathrm{m}$. (a) The gray-scale reference image obtained by scanning the dry core sample before the start of the experiments. (b) The pore-grain separated binary image obtained after performing histogram thresholding on the gray-scale reference image. Blue color represents the pore voxels. (c) The gray-scale image obtained by scanning the core during the experiment. The pore space contains two fluid phases, namely, oil and brine that is doped with 7.5 wt \% NaI. (d) The labeled image obtained after the segmentation of image (c). Blue, red, and gray colors represent brine, oil and grain voxels, respectively. 


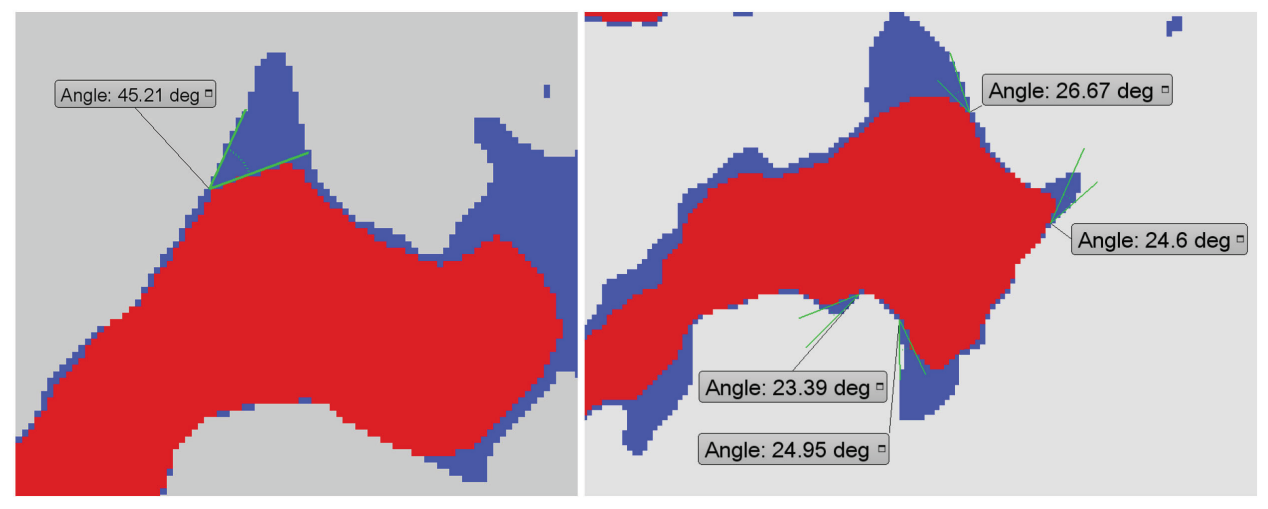

Figure 9: In-situ measurements of brine-oil contact angle in pore corners in the Berea sandstone core sample. Blue, red, and gray colors represent the brine, oil, and grain voxels, respectively. The green lines are tangents drawn to the brine-oil interface and solid surface at triple contact points. The angles created by the green lines measured through brine are brine-oil contact angles at those locations. 

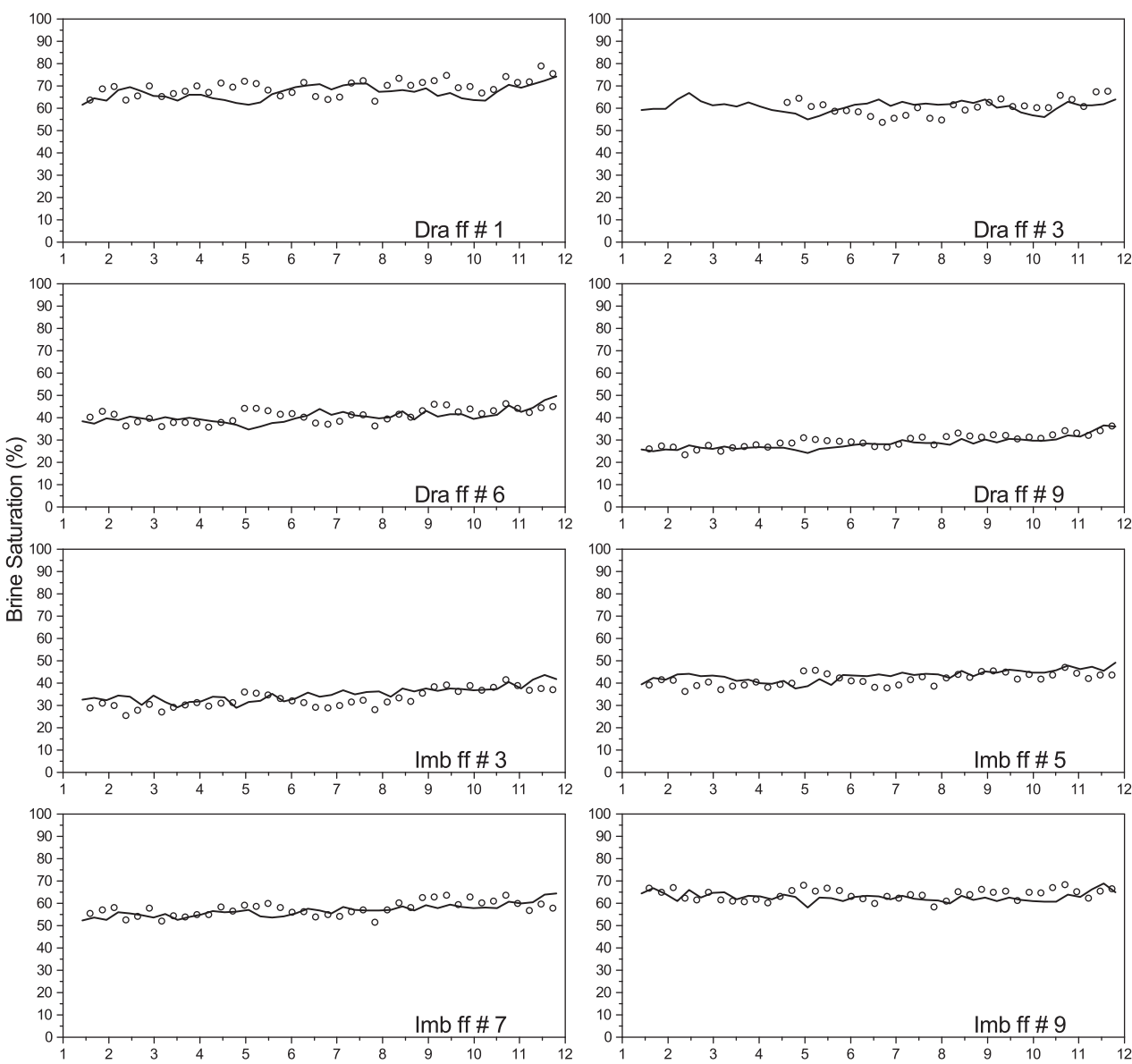

- Measured - Predicted

Position along Core $(\mathrm{mm})$

Figure 10: Experiment 1 and Simulation 1 brine saturation profiles at the end of selected fractional flows. Dra, Imb, and ff stand for drainage, imbibition, and fractional flow, respectively. The measured data from Experiment 1 are shown with empty circles, while the predicted data from Simulation 1 are shown with solid lines. The first one-third of the micro-CT slices obtained during Experiment 1 in fractional flow number 3 were corrupted due to technical problems during image acquisition. 


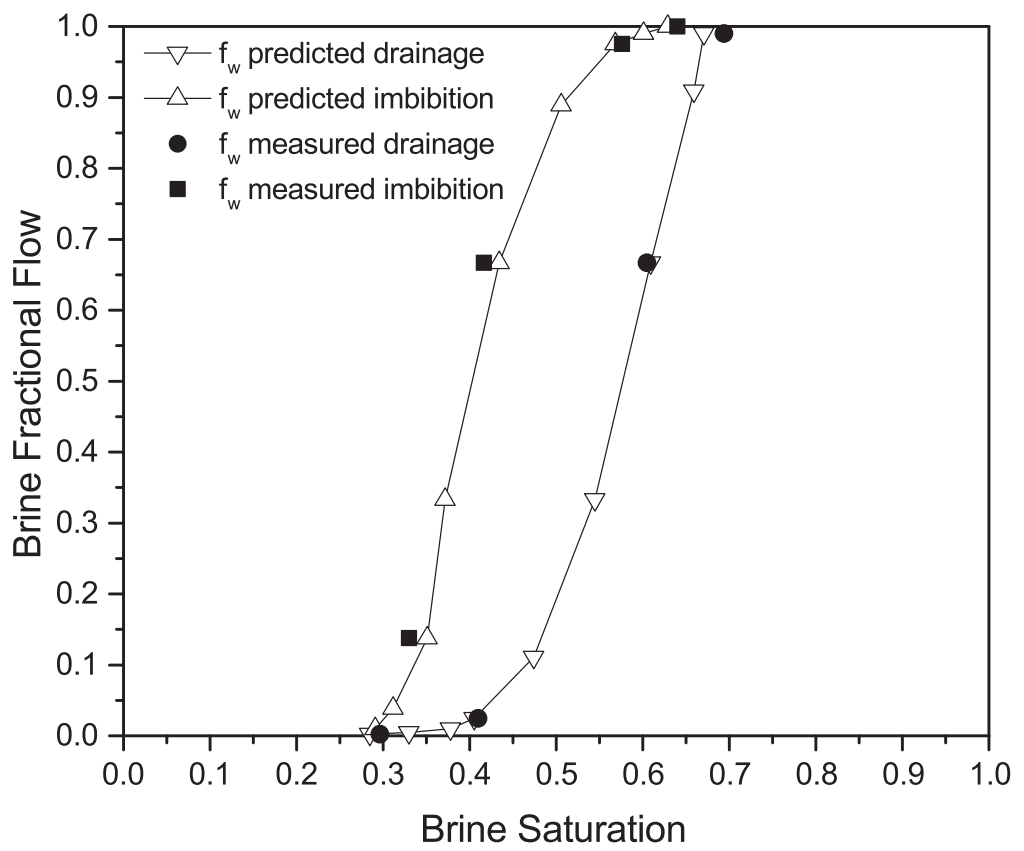

Figure 11: Fractional flow curves obtained from Experiment 1 and Simulation 1. The measured data from Experiment 1 during drainage and imbibition are shown with circles and squares, respectively. The predicted data from Simulation 1 are shown with triangles connected by solid lines. 

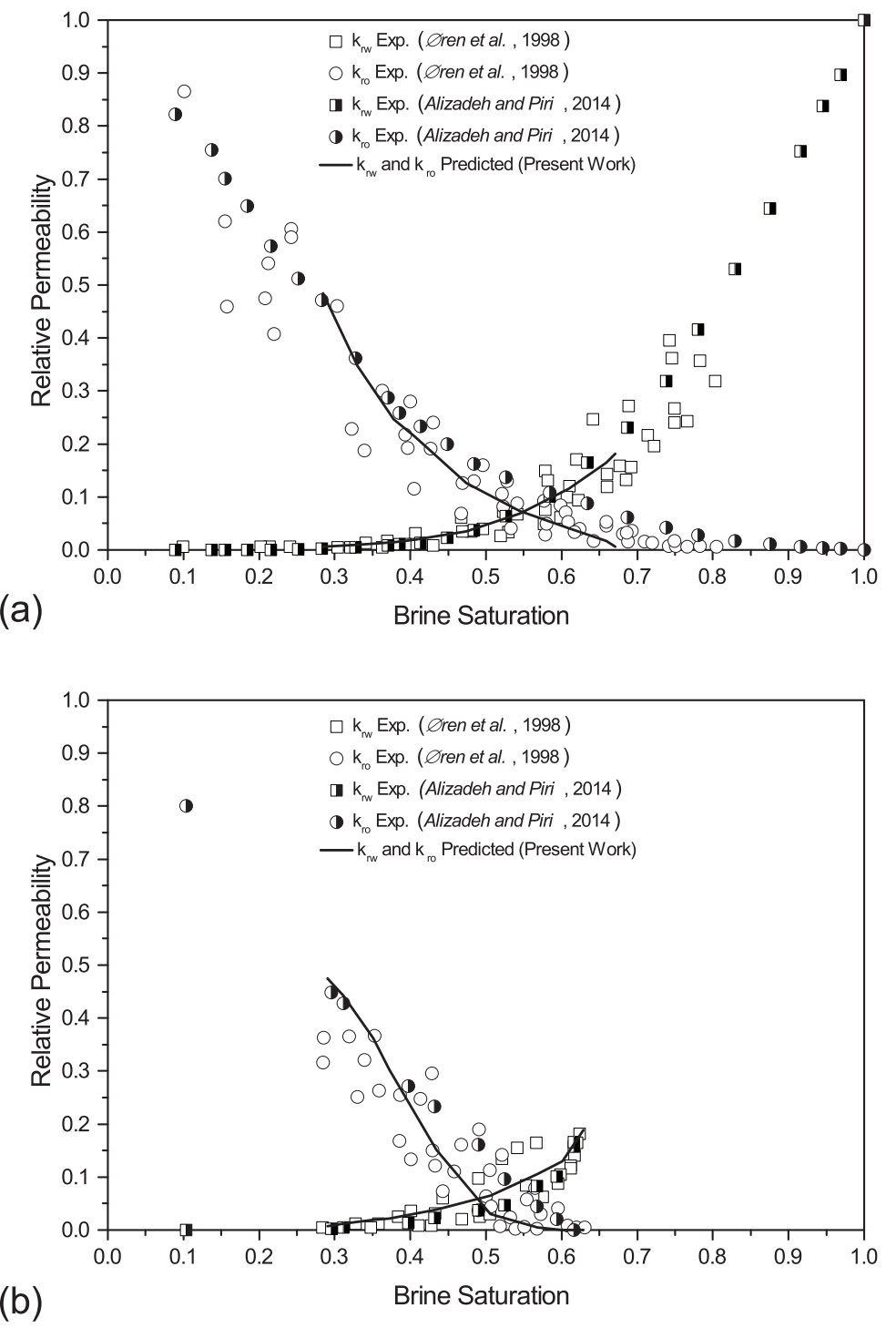

Figure 12: Bentheimer sandstone two-phase relative permeabilities versus brine saturation. Predicted data from Simulation 1 shown by solid line are compared against experimentally measured data from the literature (Øren et al., 1998; Alizadeh and Piri, 2014) shown by circles and squares. (a) Drainage. (b) Imbibition. 

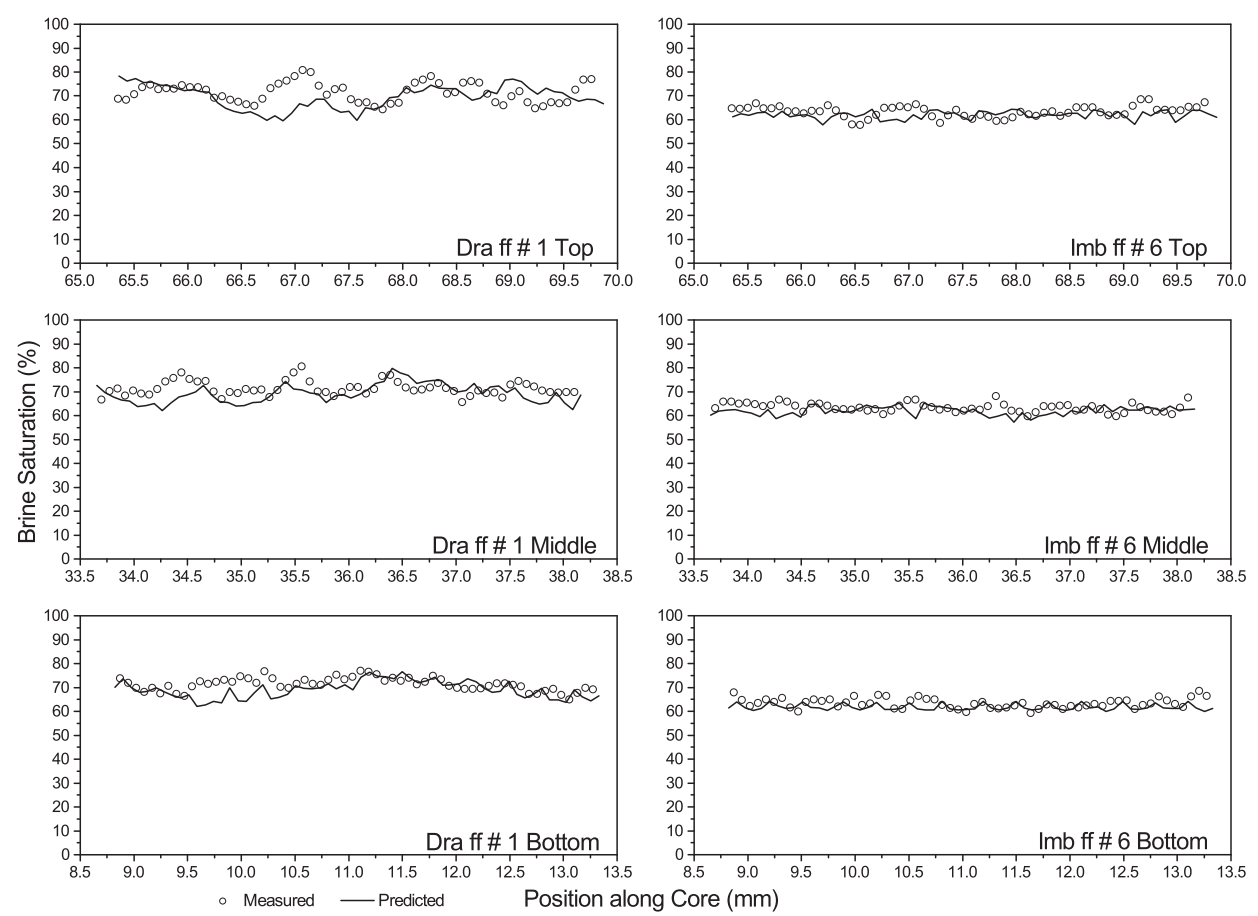

Figure 13: Experiment 2 and Simulation 2 brine saturation profiles in the bottom, middle, and top of the medium at the end of first drainage and last imbibition fractional flows. 

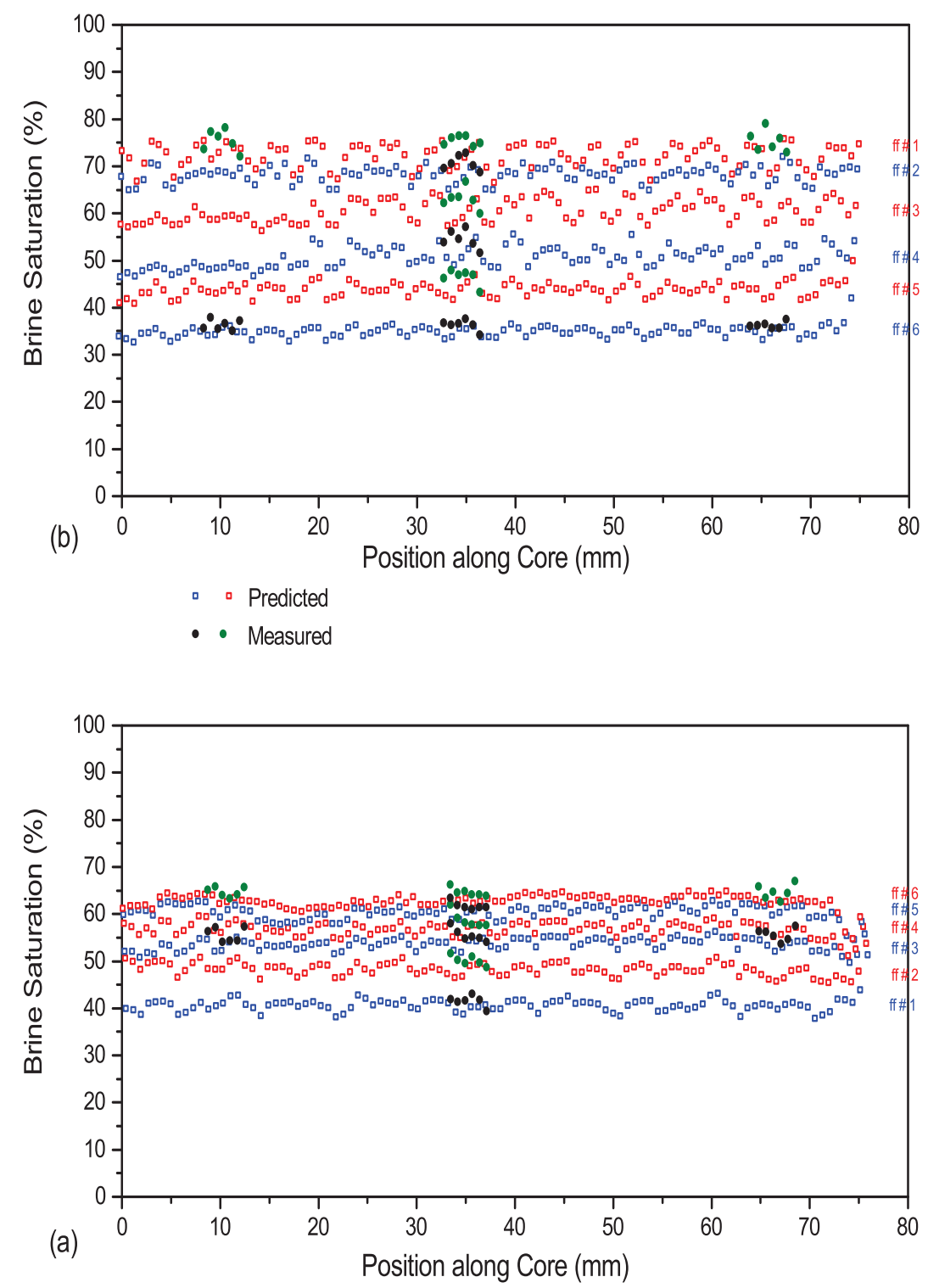

Figure 14: Experiment 2 and Simulation 2 brine saturation profiles in full length of the medium. (a) Drainage. (b) Imbibition 


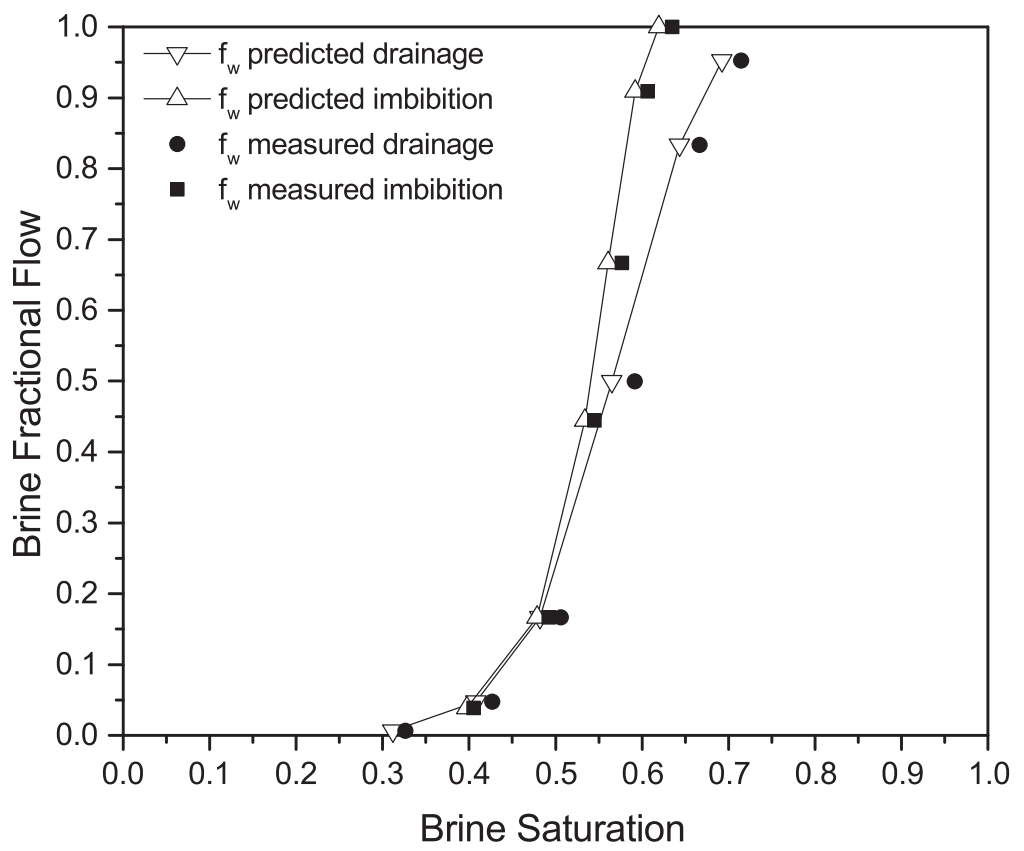

Figure 15: Fractional flow curves obtained from Experiment 2 and Simulation 2. The measured data from Experiment 2 during drainage and imbibition are shown with circles and squares, respectively. The predicted data from Simulation 2 are shown with triangles connected by solid lines. 

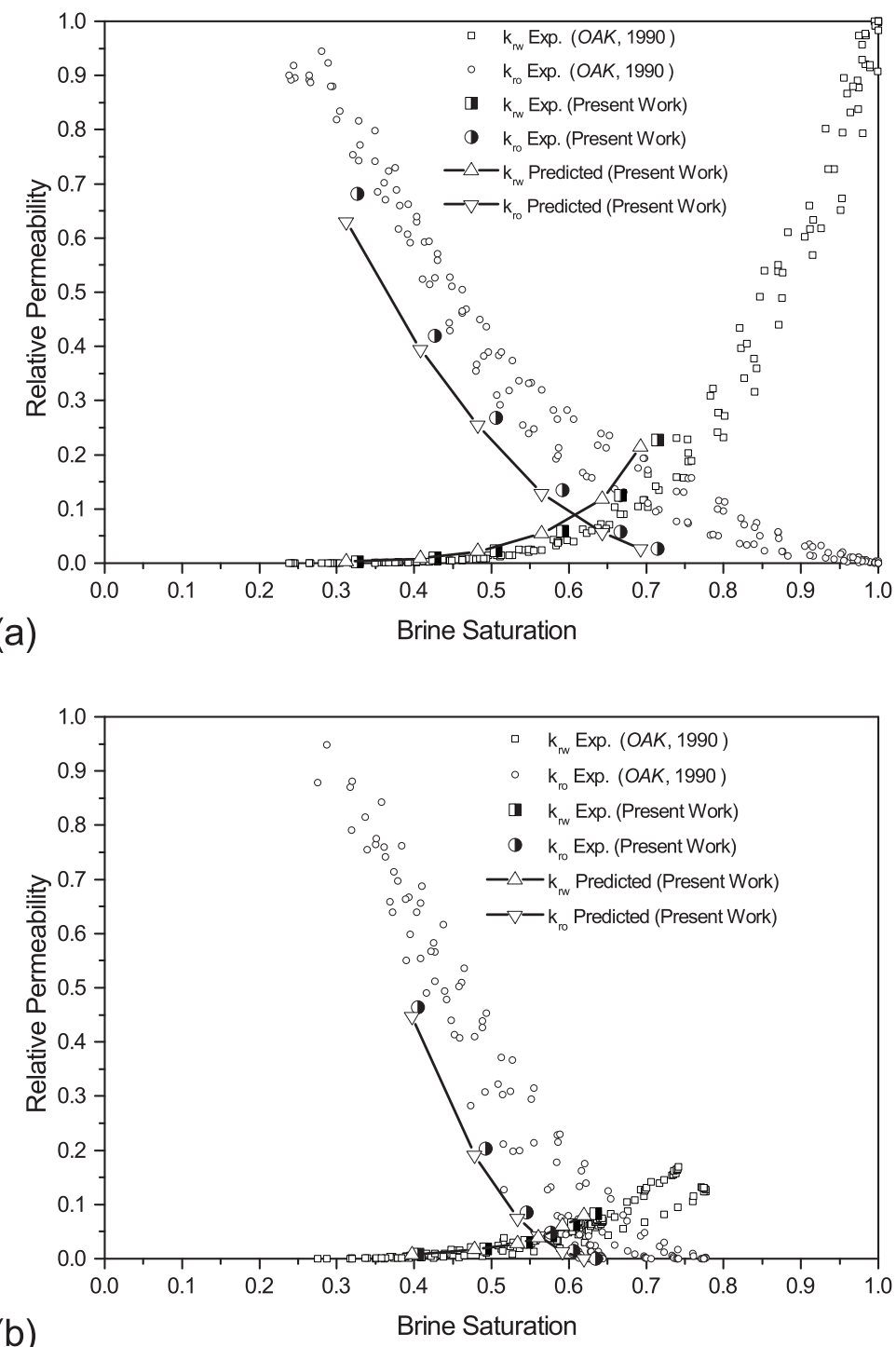

Figure 16: Berea sandstone relative permeabilities versus brine saturation. The predicted data from Simulation 2 are compared against the measured data from Experiment 2 and the experimental data by Oak (1990). (a) Drainage. (b) Imbibition. 


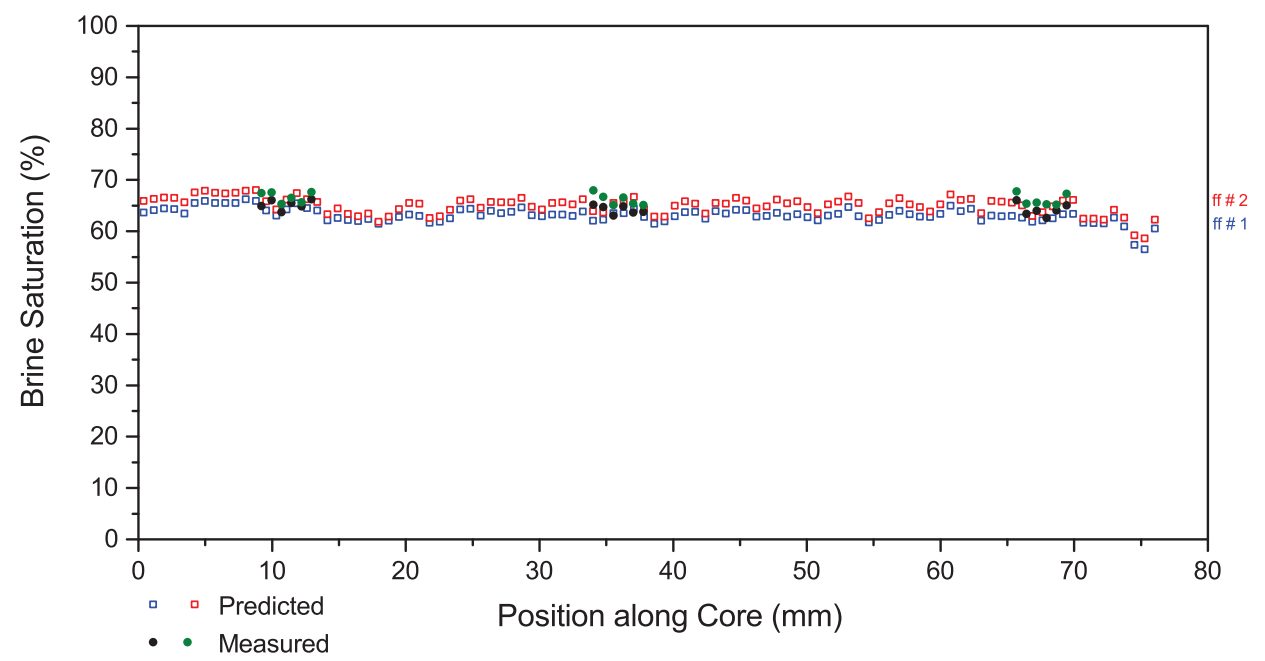

Figure 17: Experiment 3 and Simulation 3 brine saturation profiles in full length of the medium in imbibition fractional flows. 

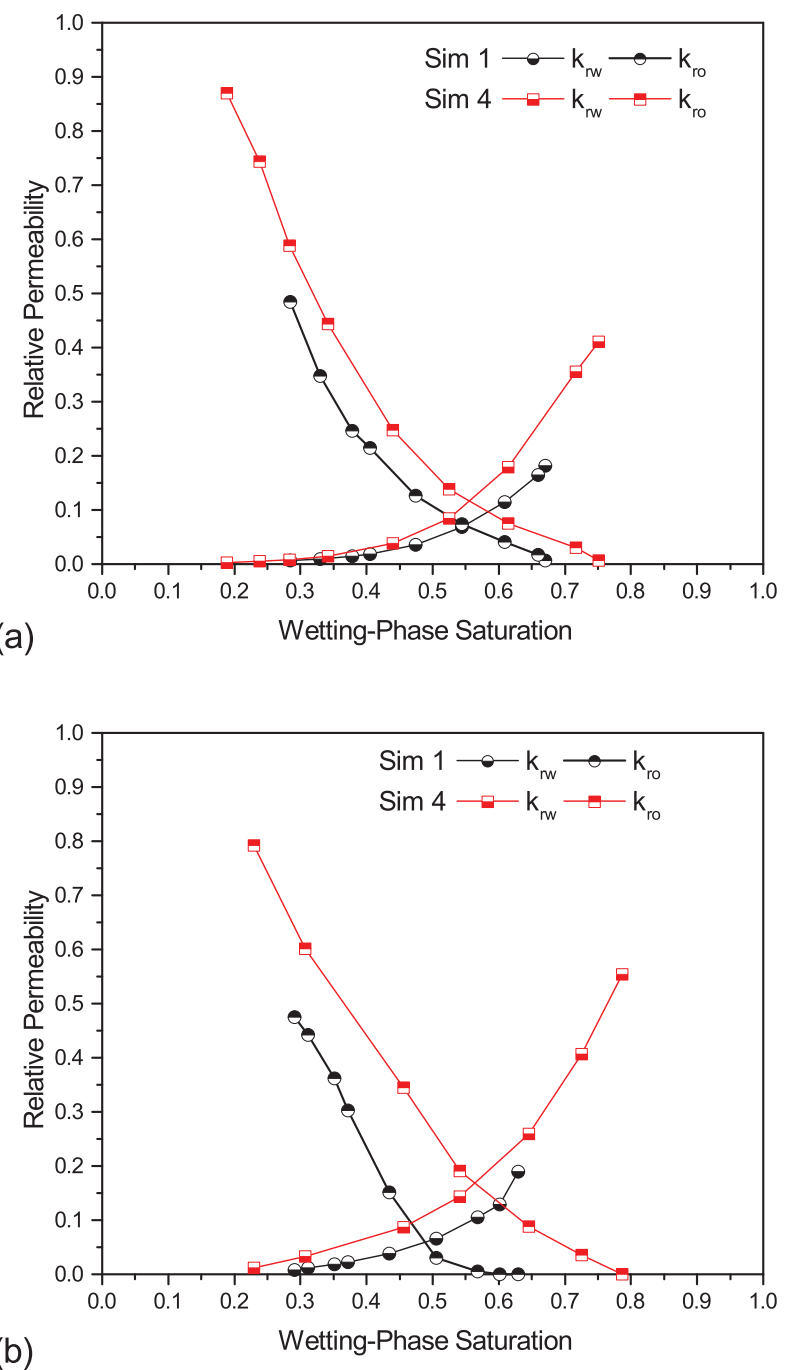

Figure 18: The effect of IFT on relative permeabilities of Bentheimer sandstone shown by comparing the results from Simulations 1 and 4. (a) Drainage. (b) Imbibition. IFT values in Simulations 1 and 4 were $46.34 \mathrm{mN} / \mathrm{m}$ and $1.0 \mathrm{mN} / \mathrm{m}$, respectively. 

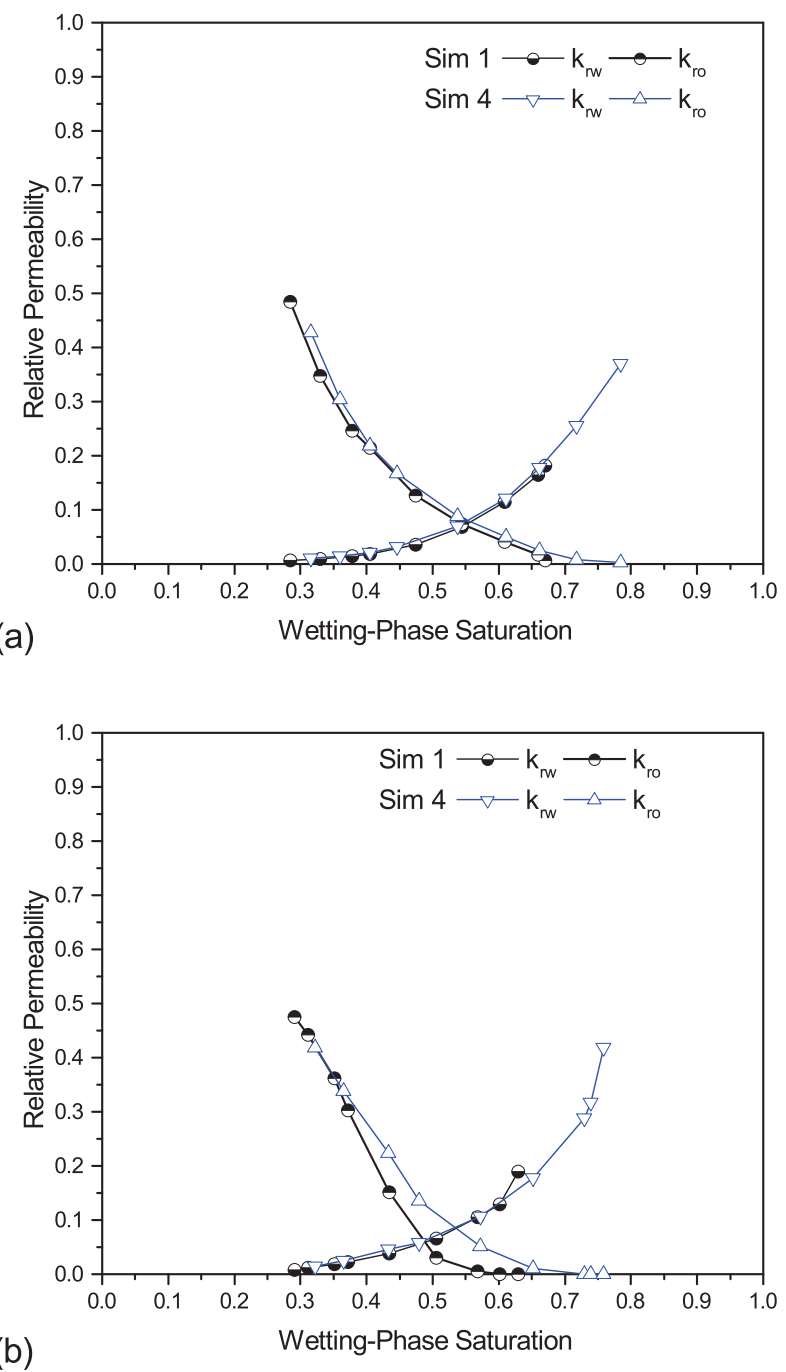

Figure 19: The effect of viscosity ratio on relative permeabilities of Bentheimer sandstone shown by comparing the results from Simulations 1 and 5. (a) Drainage. (b) Imbibition. Wetting-phase-to-oil viscosity ratio in Simulations 1 and 5 were 1.31 and 5.0, respectively. 


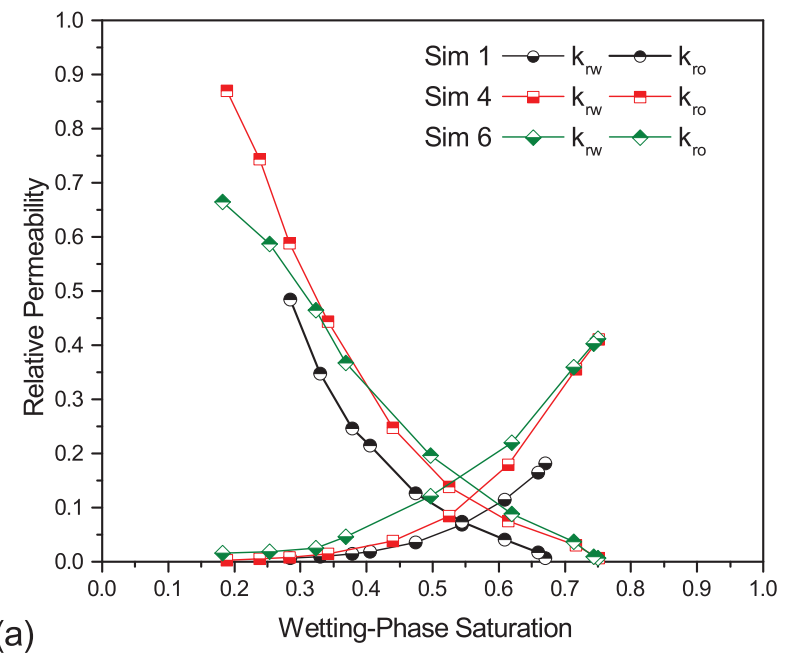

(a)

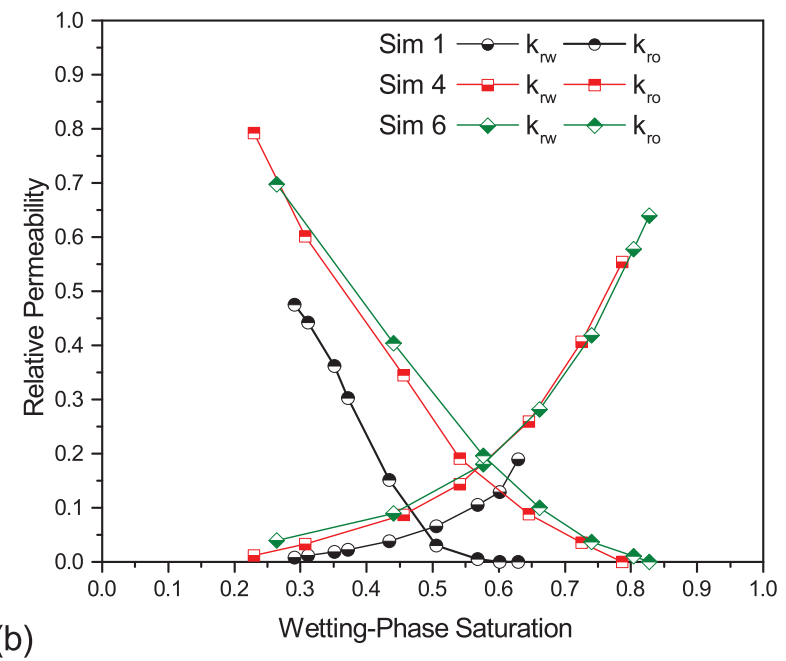

Figure 20: The combined effect of IFT and viscosity ratio on relative permeabilities of Bentheimer sandstone shown by comparing the results from Simulations 1, 4, and 6 . (a) Drainage. (b) Imbibition. IFT values and wetting-phase-to-oil viscosity ratios in Simulations 1, 4, and 6 were $46.34 \mathrm{mN} / \mathrm{m}$ and $1.31,1.0 \mathrm{mN} / \mathrm{m}$ and 1.31 , and $1.0 \mathrm{mN} / \mathrm{m}$ and 5.0, respectively. 

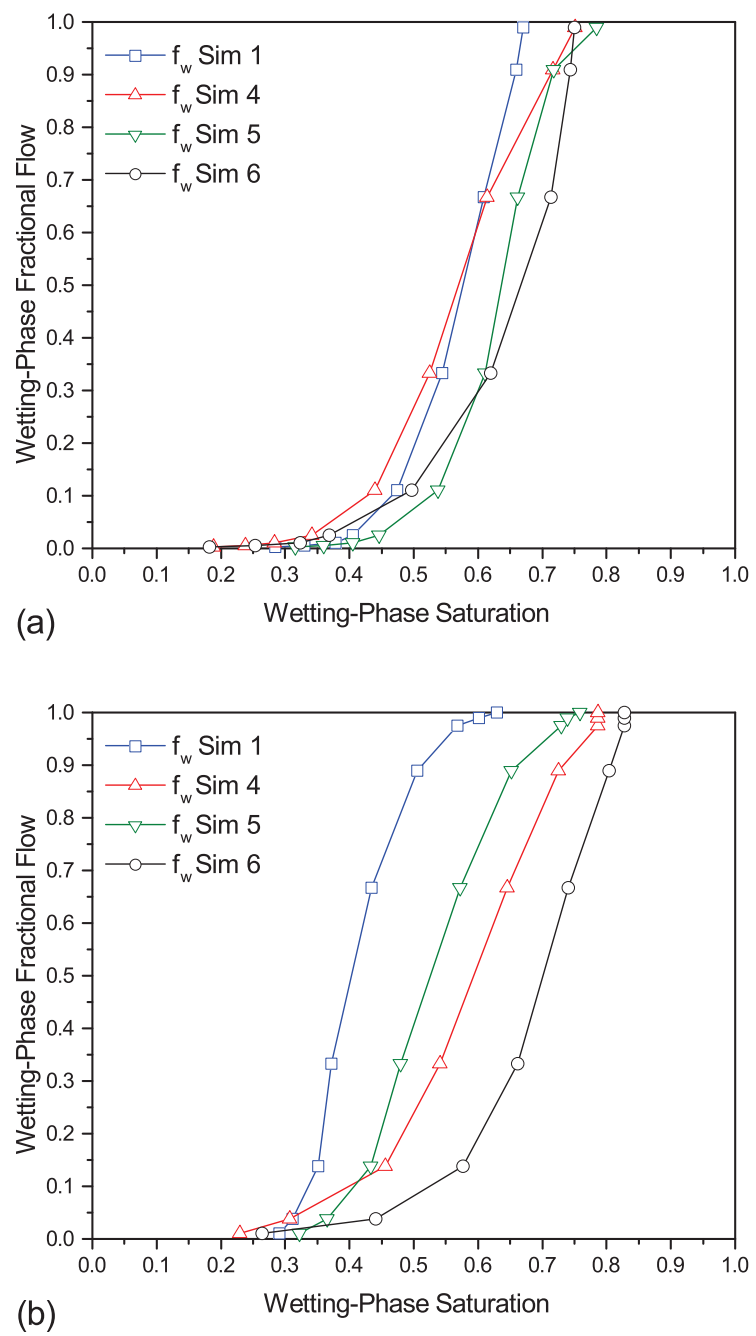

Figure 21: Wetting-phase fractional flow versus saturation obtained from Simulations 1, 4, 5, and 6. (a) Drainage. (b) Imbibition. IFT values and wetting-phase-to-oil viscosity ratios in Simulations 1, 4, 5, and 6 were $46.34 \mathrm{mN} / \mathrm{m}$ and 1.31, $1.0 \mathrm{mN} / \mathrm{m}$ and 1.31, 46.34 $\mathrm{mN} / \mathrm{m}$ and 5.0 , and $1.0 \mathrm{mN} / \mathrm{m}$ and 5.0 , respectively. 
Table 1: Previously-developed dynamic pore network models

\begin{tabular}{|c|c|c|c|}
\hline Study & $\begin{array}{l}\text { Largest } \\
\text { network size }\end{array}$ & $\begin{array}{c}\text { Phenomena } \\
\text { studied }\end{array}$ & $\begin{array}{l}\text { Validation } \\
\text { techniques }\end{array}$ \\
\hline Koplik and Lasseter (1985) & 100 & $\begin{array}{l}\text { Effect of } \mathrm{N}_{c} \\
\text { on trapping }\end{array}$ & $\mathrm{N} / \mathrm{A}$ \\
\hline Lenormand et al. (1988) & 10,000 & Flow regimes & Micro-models \\
\hline Blunt and King (1991) & 80,000 & Drainage $\mathrm{K}_{r}$ & Buckley-Leverett \\
\hline Lee et al. (1995) & 524,288 & $\begin{array}{c}\text { Imbibition } \mathrm{K}_{r}, \\
\mathrm{~S}_{o r}, \mathrm{P}_{c}\end{array}$ & $\mathrm{~N} / \mathrm{A}$ \\
\hline Kamath et al. (1996) & 262,144 & $\begin{array}{l}\text { Saturation profiles, } \\
\text { recoveries }\end{array}$ & $\begin{array}{l}\text { Unsuccessful } \\
\text { core-flooding }\end{array}$ \\
\hline Xu et al. (1999) & 131,072 & $\begin{array}{l}\text { Saturation profiles, } \\
\text { recoveries }\end{array}$ & $\begin{array}{c}\text { Recoveries in } \\
\text { miscible flooding }\end{array}$ \\
\hline van der Marck et al. (1997) & 2,401 & $\begin{array}{l}\text { Pressure field } \\
\text { in drainage }\end{array}$ & $\begin{array}{c}\text { Micro-models } \\
\text { viscosity ratio of one }\end{array}$ \\
\hline Mogensen and Stenby (1998) & 3,375 & $\begin{array}{l}\text { Trapping, } \\
\text { Sor }_{\text {or }}\end{array}$ & $\mathrm{N} / \mathrm{A}$ \\
\hline Aker et al. (1998) & 4,800 & $\begin{array}{l}\text { Pressure field } \\
\text { in drainage }\end{array}$ & $\begin{array}{c}\text { Glass beads } \\
\text { viscosity ratio of one }\end{array}$ \\
\hline Dahle and Celia (1999) & 8,381 & $\begin{array}{c}\mathrm{P}_{c} \\
\text { in drainage }\end{array}$ & $\mathrm{N} / \mathrm{A}$ \\
\hline Hughes and Blunt (2000) & 16,384 & $\begin{array}{c}\text { Kr } \\
\text { flow patterns }\end{array}$ & Micro-models \\
\hline
\end{tabular}

${ }^{\mathrm{a}}$ Maximum number of pores 
Table 2: Previously-developed dynamic pore network models (cont'd)

\begin{tabular}{|c|c|c|c|}
\hline Study & $\begin{array}{c}\text { Largest } \\
\text { network size }\end{array}$ & $\begin{array}{l}\text { Phenomena } \\
\text { studied }\end{array}$ & $\begin{array}{l}\text { Validation } \\
\text { techniques }\end{array}$ \\
\hline Constantinides and Payatakes (2000) & 6,000 & $\begin{array}{l}\text { Wetting layers, } \\
\mathrm{S}_{\text {or }}\end{array}$ & $\mathrm{N} / \mathrm{A}$ \\
\hline Thompson (2002) & 1,728 & $\begin{array}{l}\text { Imbibition } \\
\text { flow patterns }\end{array}$ & $\mathrm{N} / \mathrm{A}$ \\
\hline Singh and Mohanty (2003) & 1,920 & $\begin{array}{l}\text { Saturation profiles, } \\
\mathrm{K}_{r} \text { in drainage }\end{array}$ & $\begin{array}{c}\text { Qualitative } \\
\text { du Prey (1973) }\end{array}$ \\
\hline Nordhaug et al. (2003) & 5,000 & $\begin{array}{l}\text { Interfacial } \\
\text { velocities }\end{array}$ & $\begin{array}{c}\text { Sand pack } \\
\text { Schaefer et al. (2000) }\end{array}$ \\
\hline Løvoll et al. (2005) & 12,800 & $\begin{array}{l}\text { Effects of viscosity, } \\
\text { gravity in drainage }\end{array}$ & $\begin{array}{l}\text { Glass beads } \\
\text { tests }\end{array}$ \\
\hline Al-Gharbi and Blunt (2005) & 900 & $\begin{array}{l}\text { Displacement patterns } \\
\text { in drainage }\end{array}$ & $\mathrm{N} / \mathrm{A}$ \\
\hline Nguyen et al. (2006) & 12,349 & $\begin{array}{l}\text { Imbibition } \mathrm{K}_{r} \\
\mathrm{~S}_{\text {or }}\end{array}$ & $\begin{array}{c}\text { Oak (1990) } \\
\text { Chatzis and Morrow (1984) }\end{array}$ \\
\hline DiCarlo (2006) & 12,349 & $\begin{array}{l}\text { Saturation } \\
\text { overshoot }\end{array}$ & DiCarlo (2004) \\
\hline Piri and Karpyn (2007) & 20,890 & $\begin{array}{l}\text { Two-phase flow } \\
\text { in fracture }\end{array}$ & $\begin{array}{c}\text { Fluid occupancy } \\
\text { Karpyn and Piri (2007) }\end{array}$ \\
\hline Joekar-Niasar et al. (2010) & 42,875 & $\begin{array}{l}\text { Non-equilibrium } \\
\text { capillarity theory }\end{array}$ & $\begin{array}{c}\text { Theoretical } \\
\text { Hassanizadeh and Gray (1990) }\end{array}$ \\
\hline Tørå et al. (2012) & 767 & $\begin{array}{l}\text { Saturation profiles, } \\
\text { resistivity index }\end{array}$ & $\begin{array}{l}\text { Sandpack } \\
\text { tests }\end{array}$ \\
\hline Sheng and Thompson (2013) & 1,532 & $\begin{array}{l}\text { Coupling with } \\
\text { reservoir simulator }\end{array}$ & $\mathrm{N} / \mathrm{A}$ \\
\hline
\end{tabular}

${ }^{\mathrm{a}}$ Maximum number of pores 
Table 3: Properties of the Pore Network Representative of the Bentheimer Sandstone Core Sample Used in Experiment 1

\begin{tabular}{cccc}
\hline \hline Item & Throats & Pores & Total \\
\hline Number & 306,825 & 131,346 & 438,171 \\
Porosity excl. clay (\%) & 9.697 & 13.631 & 23.328 \\
Porosity incl. clay (\%) & 11.261 & 13.772 & 25.033 \\
Absolute Permeability (mD) & & & 2716 \\
Length (mm) & & & 12.977 \\
Diameter (mm) & & & 4.972 \\
Minimum coordination number & & 150 & \\
Maximum coordination number & & 4.622 & \\
Average coordination number & & 0.30 & 0.28 \\
Minimum radius $(\mu \mathrm{m})$ & 0.28 & 148.48 & 148.48 \\
Maximum radius $(\mu \mathrm{m})$ & 101.00 & 0.376 & 0.546 \\
Average radius $(\mu \mathrm{m})$ & 13.71 & 25.96 & 17.38 \\
Average shape factor & 0.042 & 0.046 & 0.044 \\
Triangular cross sections $(\%)$ & 79.790 & 70.665 & 77.055 \\
Square cross sections $(\%)$ & 19.590 & 28.959 & 22.399 \\
Circular cross sections $(\%)$ & 0.620 & 0.3248 \\
Connected to the inlet & 3363 & 0 & 589 \\
Connected to the outlet & 3248 & 0 & \\
Isolated clusters & & & \\
\hline \hline
\end{tabular}


Table 4: Properties of the Pore Network Representative of the Berea Sandstone Core Sample Used in Experiments 2 and 3

\begin{tabular}{|c|c|c|c|}
\hline Item & Throats & Pores & Total \\
\hline Number & $3,913,222$ & $1,891,260$ & $5,804,482$ \\
\hline Porosity excl. clay (\%) & 8.236 & 12.616 & 20.852 \\
\hline Porosity incl. clay (\%) & 8.458 & 12.892 & 21.350 \\
\hline Absolute Permeability (mD) & & & 638 \\
\hline Length (mm) & & & 76.4 \\
\hline Diameter (mm) & & & 4.58 \\
\hline Minimum coordination number & & 0 & \\
\hline Maximum coordination number & & 45 & \\
\hline Average coordination number & & 4.017 & \\
\hline Minimum radius $(\mu \mathrm{m})$ & 0.21 & 0.25 & 0.21 \\
\hline Maximum radius $(\mu \mathrm{m})$ & 63.50 & 101.0 & 101.0 \\
\hline Average radius $(\mu \mathrm{m})$ & 6.95 & 13.95 & 9.27 \\
\hline Average shape factor & 0.039 & 0.047 & 0.042 \\
\hline Triangular cross sections (\%) & 78.522 & 57.667 & 71.592 \\
\hline Square cross sections (\%) & 20.954 & 41.594 & 27.813 \\
\hline Circular cross sections (\%) & 0.524 & 0.739 & 0.595 \\
\hline Connected to the inlet & 1515 & 0 & 1515 \\
\hline Connected to the outlet & 1507 & 0 & 1507 \\
\hline Isolated clusters & & & 24 \\
\hline Isolated & 7460 & 7791 & 15251 \\
\hline
\end{tabular}

Table 5: Number of Experiments and Their Conditions and Procedures

\begin{tabular}{ccccccc}
\hline $\begin{array}{c}\text { Experiment } \\
\text { no. }\end{array}$ & $\begin{array}{c}\text { Core } \\
\text { sample }\end{array}$ & $\begin{array}{c}\text { Temp. }\left({ }^{\circ} \mathrm{C}\right) / \\
\text { Press. }(\mathrm{MPa})\end{array}$ & $\begin{array}{c}\text { Confining } \\
\text { press. }(\mathrm{MPa})\end{array}$ & $\begin{array}{c}\text { Wetting } \\
\text { phase }\end{array}$ & $\begin{array}{c}\text { Non-wetting } \\
\text { phase }\end{array}$ & $\begin{array}{c}\text { Experimental } \\
\text { procedure }\end{array}$ \\
\hline 1 & $\begin{array}{c}\text { Bentheimer } \\
\text { sandstone }\end{array}$ & $24 / 2.76$ & 4.83 & $\begin{array}{c}\text { Brine } \\
1.5 \text { wt. } \% \text { NaI }\end{array}$ & n-Decane & Steady state \\
\hline 2 & $\begin{array}{c}\text { Berea } \\
\text { sandstone }\end{array}$ & $24 / 0.90$ & 1.52 & Brine & Soltrol & Steady state \\
\hline 3 & Berea & $24 / 0.90$ & 1.52 & Brine & Soltrol & Unsteady state \\
\hline \hline
\end{tabular}


Table 6: Dimensions and Petrophysical Properties of the Core Samples Used in the Ex-

\begin{tabular}{|c|c|c|c|c|c|c|}
\hline $\begin{array}{c}\text { Experiment } \\
\text { no. }\end{array}$ & $\begin{array}{c}\text { Core } \\
\text { sample }\end{array}$ & $\begin{array}{c}\text { Diameter } \\
\mathrm{mm}\end{array}$ & $\begin{array}{l}\text { Length } \\
\text { mm }\end{array}$ & $\begin{array}{c}\phi^{\mathrm{a}} \\
\%\end{array}$ & $\begin{array}{c}\mathrm{K}_{\mathrm{abs}} \\
\mathrm{mD}\end{array}$ & $\begin{array}{c}\text { Pore volume } \\
\mathrm{mm}^{3}\end{array}$ \\
\hline 1 & $\begin{array}{l}\text { Bentheimer } \\
\text { sandstone }\end{array}$ & 4.97 & 12.98 & 23.42 & $2568^{\mathrm{b}}$ & 58.97 \\
\hline 2,3 & $\begin{array}{c}\text { Berea } \\
\text { sandstone }\end{array}$ & 4.58 & 76.40 & 21.69 & $623^{\mathrm{c}}$ & 273.01 \\
\hline
\end{tabular}

${ }^{\mathrm{a}}$ Helium porosity

${ }^{\mathrm{b}}$ Klinkenberg-corrected helium permeability

${ }^{\mathrm{c}}$ Brine permeability

Table 7: Properties of the Fluids Used in the Experiments Measured at Experiment Conditions

\begin{tabular}{ccccc}
\hline $\begin{array}{c}\text { Experiment } \\
\text { no. }\end{array}$ & Fluid & $\begin{array}{c}\text { Viscosity } \\
\mathrm{cP}\end{array}$ & $\begin{array}{c}\text { Density } \\
\mathrm{gr} / \mathrm{cm}^{3}\end{array}$ & $\begin{array}{c}\mathrm{IFT}^{\mathrm{a}} \\
\mathrm{mN} / \mathrm{m}\end{array}$ \\
\hline \multirow{4}{*}{$\begin{array}{c}\text { n-Decane } \\
\text { Brine }\end{array}$} & 0.942 & 0.727 & \\
& 1.5 wt \% NaI & 1.237 & 1.009 & 46.34 \\
\hline \multirow{4}{*}{$\begin{array}{c}\text { Soltrol } \\
170\end{array}$} & 2.851 & 0.774 & \\
& Brine & & & 41.33 \\
& 7.5 wt \% NaI & 1.240 & 1.055 & \\
\hline
\end{tabular}

${ }^{a}$ Brine-Oil interfacial tension 
Table 8: Brine and Oil Flow Rates, Capillary Numbers, and Scanned Spots in Experiment $\underline{1}$

\begin{tabular}{|c|c|c|c|c|c|c|}
\hline & $\begin{array}{c}\mathrm{FF} \\
\text { no. }^{\mathrm{a}}\end{array}$ & $f_{\text {brine }}{ }^{b}$ & $\begin{array}{c}\text { Qbrine } \\
\mathrm{cm}^{3} / \mathrm{min}\end{array}$ & $\begin{array}{c}Q_{\text {oil }} \\
\mathrm{cm}^{3} / \min \end{array}$ & $\mathrm{N}_{c}$ & $\begin{array}{c}\text { Scanned } \\
\text { spots }\end{array}$ \\
\hline \multirow{9}{*}{ Drainage } & 1 & 0.990 & 0.1 & 0.001 & $7.45 \times 10^{-8}$ & Full \\
\hline & 2 & 0.909 & 0.05 & 0.005 & $3.72 \times 10^{-7}$ & - \\
\hline & 3 & 0.667 & 0.02 & 0.01 & $7.45 \times 10^{-7}$ & Full \\
\hline & 4 & 0.333 & 0.01 & 0.02 & $1.49 \times 10^{-6}$ & - \\
\hline & 5 & 0.111 & 0.005 & 0.04 & $2.98 \times 10^{-6}$ & - \\
\hline & 6 & 0.024 & 0.002 & 0.08 & $5.96 \times 10^{-6}$ & Full \\
\hline & 7 & 0.010 & 0.001 & 0.1 & $7.45 \times 10^{-6}$ & - \\
\hline & 8 & 0.005 & 0.001 & 0.2 & $1.49 \times 10^{-5}$ & - \\
\hline & 9 & 0.003 & 0.001 & 0.4 & $2.98 \times 10^{-5}$ & Full \\
\hline \multirow{9}{*}{ Imbibition } & 1 & 0.010 & 0.002 & 0.2 & $1.96 \times 10^{-7}$ & - \\
\hline & 2 & 0.039 & 0.004 & 0.1 & $3.91 \times 10^{-7}$ & - \\
\hline & 3 & 0.138 & 0.008 & 0.05 & $7.83 \times 10^{-7}$ & Full \\
\hline & 4 & 0.333 & 0.01 & 0.02 & $9.78 \times 10^{-7}$ & - \\
\hline & 5 & 0.667 & 0.02 & 0.01 & $1.96 \times 10^{-6}$ & Full \\
\hline & 6 & 0.889 & 0.04 & 0.005 & $3.91 \times 10^{-6}$ & - \\
\hline & 7 & 0.976 & 0.08 & 0.002 & $7.83 \times 10^{-6}$ & Full \\
\hline & 8 & 0.990 & 0.1 & 0.001 & $9.78 \times 10^{-6}$ & - \\
\hline & 9 & 1 & 0.2 & 0 & $1.96 \times 10^{-5}$ & Full \\
\hline
\end{tabular}

${ }^{\text {a Fractional flow number }}$

${ }^{\mathrm{b}}$ Fractional flow of brine 
Table 9: Brine and Oil Flow Rates, Capillary Numbers, and Scanned Spots in Experiment 2

\begin{tabular}{|c|c|c|c|c|c|c|}
\hline & $\begin{array}{c}\text { FF } \\
\text { no. }\end{array}$ & $f_{\text {brine }}{ }^{b}$ & $\begin{array}{c}Q_{\text {brine }} \\
\mathrm{cm}^{3} / \mathrm{min}\end{array}$ & $\begin{array}{c}Q_{\text {oil }} \\
\mathrm{cm}^{3} / \mathrm{min}\end{array}$ & $\mathrm{N}_{c}$ & $\begin{array}{c}\text { Scanned } \\
\text { spots }\end{array}$ \\
\hline \multirow{6}{*}{ Drainage } & 1 & 0.952 & 0.1 & 0.005 & $5.07 \times 10^{-7}$ & $1,2,3$ \\
\hline & 2 & 0.833 & 0.05 & 0.01 & $1.01 \times 10^{-6}$ & 2 \\
\hline & 3 & 0.5 & 0.02 & 0.02 & $2.03 \times 10^{-6}$ & 2 \\
\hline & 4 & 0.167 & 0.01 & 0.05 & $5.07 \times 10^{-6}$ & 2 \\
\hline & 5 & 0.048 & 0.005 & 0.1 & $1.01 \times 10^{-5}$ & 2 \\
\hline & 6 & 0.007 & 0.001 & 0.15 & $1.52 \times 10^{-5}$ & $1,2,3$ \\
\hline \multirow{6}{*}{ Imbibition } & 1 & 0.038 & 0.002 & 0.05 & $2.40 \times 10^{-7}$ & 2 \\
\hline & 2 & 0.167 & 0.004 & 0.02 & $4.80 \times 10^{-7}$ & 2 \\
\hline & 3 & 0.444 & 0.008 & 0.01 & $9.60 \times 10^{-7}$ & $1,2,3$ \\
\hline & 4 & 0.667 & 0.01 & 0.005 & $1.20 \times 10^{-6}$ & 2 \\
\hline & 5 & 0.909 & 0.02 & 0.002 & $2.40 \times 10^{-6}$ & 2 \\
\hline & 6 & 1 & 0.05 & 0 & $6.00 \times 10^{-6}$ & $1,2,3$ \\
\hline
\end{tabular}

Fractional flow number

${ }^{\mathrm{b}}$ Fractional flow of brine

Table 10: Brine and Oil Flow Rates, Capillary Numbers, and Scanned Spots in Experiment $\underline{\underline{3}}$

\begin{tabular}{cccccc}
\hline 3 & \multicolumn{6}{c}{$\begin{array}{l}\text { FF } \\
\text { no. }^{\mathrm{a}}\end{array}$} & $\begin{array}{c}\text { Qbrine } \\
\mathrm{cm}^{3} / \mathrm{min}\end{array}$ & $\begin{array}{c}\text { Qoil } \\
\mathrm{cm}^{3} / \mathrm{min}\end{array}$ & $\mathrm{N}_{c}$ & $\begin{array}{c}\text { Scanned } \\
\text { spots }\end{array}$ \\
\hline Drainage & 1 & 0.001 & 0.15 & $1.52 \times 10^{-5}$ & $1,2,3$ \\
& & & & & \\
Imbibition & 1 & 0.1 & 0 & $1.20 \times 10^{-5}$ & $1,2,3$ \\
& 2 & 0.15 & 0 & $1.80 \times 10^{-5}$ & $1,2,3$ \\
\hline \hline a Fractional flow number
\end{tabular}

a Fractional flow number

Table 11: Scanned Spots Locations Along the Berea Core Sample in Experiments 2 and 3

\begin{tabular}{ccc}
\hline \hline Spot no. & $\begin{array}{c}\text { Middle of spot } \\
\text { from inlet } \mathrm{mm}\end{array}$ & $\begin{array}{c}\text { Spot length } \\
\mathrm{mm}\end{array}$ \\
\hline 1 & 11.07 & 4.41 \\
2 & 35.90 & 4.41 \\
3 & 67.55 & 4.41 \\
\hline \hline
\end{tabular}


Table 12: In-Situ Brine-Oil Contact Angles Measured in the Bentheimer and Berea Sandstone Samples During Experiments and Used in Simulations

\begin{tabular}{cccccc}
\hline \hline Core & Exp/ & Process $/$ & Average & Min & Max \\
sample & Sim no. & Type & $\theta_{\text {AM }}$ & $\theta_{\text {AM }}$ & $\theta_{\text {AM }}$ \\
\hline \multirow{2}{*}{ Bentheimer } & \multirow{2}{*}{ Exp 1 } & Drainage & 22.83 & 10.81 & 32.33 \\
sandstone & \multirow{2}{*}{ Sim 1 } & Imbibition & 28.22 & 19.83 & 42.11 \\
& & Receding & 22 & 12 & 32 \\
& \multirow{2}{*}{ Exp 2,3 } & Advancing & 45 & 35 & 55 \\
& & Drainage & 15.51 & 6.37 & 26.57 \\
sandstone & \multirow{2}{*}{ Sim 2,3 } & Receding & 16 & 6 & 26 \\
& & Advancing & 50 & 40 & 60 \\
\hline \hline
\end{tabular}

Table 13: Properties of the Fluids and Ranges of the Capillary Number in Simulations 4, 5 , and 6

\begin{tabular}{|c|c|c|c|c|c|c|}
\hline $\begin{array}{c}\text { Simulation } \\
\text { no. }\end{array}$ & Fluid & $\begin{array}{c}\text { Viscosity } \\
\mathrm{cP}\end{array}$ & $\begin{array}{l}\text { Density } \\
\mathrm{gr} / \mathrm{cm}^{3}\end{array}$ & $\begin{array}{c}\mathrm{IFT} \\
\mathrm{mN} / \mathrm{m}\end{array}$ & $\begin{array}{l}\text { Drainage } \\
\mathrm{N}_{c} \text { range }\end{array}$ & $\begin{array}{l}\text { Imbibition } \\
\mathrm{N}_{c} \text { range }\end{array}$ \\
\hline \multirow{2}{*}{4} & $\mathrm{NWP}^{\mathrm{a}}$ & & & \multirow{2}{*}{1.0} & \multirow{2}{*}{$3 \times 10^{-6}-1 \times 10^{-3}$} & \multirow{2}{*}{$9 \times 10^{-6}-9 \times 10^{-4}$} \\
\hline & $\mathrm{WP}^{\mathrm{b}}$ & 1.237 & 1.009 & & & \\
\hline \multirow{2}{*}{5} & NWP & 1.0 & 0.727 & \multirow{2}{*}{46.34} & \multirow{2}{*}{$8 \times 10^{-8}-3 \times 10^{-5}$} & \multirow{2}{*}{$8 \times 10^{-7}-8 \times 10^{-5}$} \\
\hline & WP & 5.0 & 1.009 & & & \\
\hline \multirow{2}{*}{6} & NWP & 1.0 & 0.727 & \multirow{2}{*}{1.0} & \multirow{2}{*}{$4 \times 10^{-6}-1 \times 10^{-3}$} & \multirow{2}{*}{$4 \times 10^{-5}-4 \times 10^{-3}$} \\
\hline & WP & 5.0 & 1.009 & & & \\
\hline
\end{tabular}

a Non-wetting phase

bWetting phase 
- Dynamic pore network modeling of two-phase flow at the core-scale is presented.

- The model is used as a pore-to-core up-scaling platform.

- Miniature core-flooding experiments are performed and used to rigorously validate the model. 